\title{
OXIDATIVE STRESS IN CARDIAC HYPERTROPHY: FROM MOLECULAR MECHANISMS TO NOVEL THERAPEUTIC TARGETS
}

Chrishan J.A. Ramachandra*1,2, Shuo Cong ${ }^{1,2}$, Xavier Chan, Ping En, Fan Yu, Derek J. Hausenloy ${ }^{1-5}$

${ }^{1}$ National Heart Research Institute Singapore, National Heart Centre Singapore, Singapore ${ }^{2}$ Cardiovascular \& Metabolic Disorders Program, Duke-National University of Singapore Medical School, Singapore

${ }^{3}$ Yong Loo Lin School of Medicine, National University Singapore, Singapore ${ }^{4}$ The Hatter Cardiovascular Institute, University College London, London, UK ${ }^{5}$ Cardiovascular Research Center, College of Medical and Health Sciences, Asia University, Taiwan

Running title: Oxidative stress and cardiac hypertrophy

${ }^{*}$ Corresponding author:

Dr. Chrishan J.A. Ramachandra

National Heart Research Institute Singapore

National Heart Centre Singapore

Singapore

E-mail: chrishan.ramachandra@nhcs.com.sg 


\begin{abstract}
When faced with increased workload the heart undergoes remodelling, where it increases its muscle mass in an attempt to preserve normal function. This is referred to as cardiac hypertrophy and if sustained, can lead to impaired contractile function. Experimental evidence supports oxidative stress as a critical inducer of both genetic and acquired forms of cardiac hypertrophy, a finding which is reinforced by elevated levels of circulating oxidative stress markers in patients with cardiac hypertrophy. These observations formed the basis for using antioxidants as a therapeutic means to attenuate cardiac hypertrophy and improve clinical outcomes. However, the use of antioxidant therapies in the clinical setting has been associated with inconsistent results, despite antioxidants having been shown to exert protection in several animal models of cardiac hypertrophy. This has forced us to revaluate the mechanisms, both upstream and downstream of oxidative stress, where recent studies demonstrate that apart from conventional mediators of oxidative stress, metabolic influences as well as impairment in other cellular processes contribute to disease pathophysiology. Importantly, novel therapeutic interventions have been identified that can normalise such impairments, thereby counteracting oxidative stress with accompanying attenuation of cardiac hypertrophy and improved cardiac function. Here, we review the latest literature on these novel mechanisms and intervention strategies with the aim of better understanding the complexities of oxidative stress for more precise targeted therapeutic approaches to prevent cardiac hypertrophy.
\end{abstract}

\title{
KEYWORDS
}

Oxidative stress; Reactive oxygen species; Cardiac hypertrophy; Antioxidants; Metabolism; Inflammation 


\section{INTRODUCTION}

Cardiac hypertrophy is a condition where the heart increases its muscle mass in response to intrinsic or external stress. Though considered adaptive at first to accommodate the demands of increased cardiac workload and biomechanical stress, sustained hypertrophy can lead to maladaptive remodelling with accompanying contractile dysfunction. Cardiac hypertrophy is usually associated with enlargement of the left ventricle (LV), although enlargement of the right ventricle (RV) is not uncommon [1]. Various aetiologies have been described for cardiac hypertrophy, including pressure overload (acquired) and mutations in sarcomeric and nonsarcomeric genes (inherited), and hence it is not surprising that cardiac hypertrophy is commonly observed in the setting of hypertension, hypertrophic cardiomyopathy (HCM), valvular disease and congenital heart disease [2].

With regard to the molecular pathogenesis, oxidative stress has been identified as a key mediator of cardiac hypertrophy across several aetiologies. In support of this, when five mouse models of cardiac hypertrophy with differing aetiology were compared, six cellular processes were found to be common, including oxidative stress, aging, contraction, developmental processes, cell differentiation, and cell proliferation [3]. More recently, OXY-SCORE, a global indicator of oxidative stress that combines individual plasma markers of oxidative damage and antioxidant capacity was able to differentiate between those with and without left ventricular hypertrophy (LVH), independent of other risk factors [4]. Similarly, high plasma protein carbonyl levels (a biomarker of oxidative protein modification) have been found to be an independent predictor of eccentric LVH in chronic haemodialysis patients [5]. Moreover, when assessing the association between oxidative stress biomarkers and cardiovascular risk factors and LVH in children with chronic kidney disease, hypertension and dyslipidaemia were found to correlate with lipid oxidation while oxidised low-density lipoprotein seemed to be an important marker correlating with LVH [6]. These studies highlight oxidative stress to be a critical pathophysiological feature in this setting cardiac hypertrophy.

Oxidative stress is considered the imbalance between reactive oxygen species (ROS) formation and cellular antioxidant capacity due to enhanced ROS generation and/or dysfunction of the antioxidant system. ROS can be generated from several cellular sources, including nicotinamide adenine dinucleotide phosphate oxidase (NOX), xanthine oxidase and the mitochondrial electron transport chain [7] and for a comprehensive review of the types of species generated we would like to direct the reader to the following excellent reviews $[8,9]$. It is important to note that ROS production is a normal physiological process and is essential for redox balance, however, uncontrolled ROS overproduction has been found to induce protein and lipid peroxidation as well as DNA mutagenesis that can lead to irreversible cell damage or death [10]. In the setting of cardiac hypertrophy, oxidative stress has been found to activate ROS-sensitive pro-hypertrophic and remodelling signalling cascades [11] and consistently, treatment with antioxidants have been shown to attenuate the hypertrophic response as evidenced by reduction in cardiac remodelling and improvements in cardiac function in various animal models [12]. However, antioxidant 
treatment in the clinical setting has yielded inconsistent results [13], forcing us to revaluate the mechanisms by which oxidative stress exerts its detrimental effects.

Recently, several studies have attempted to identify novel upstream mediators of oxidative stress as well as downstream signalling pathways by which oxidative stress impacts cellular processes. Importantly, these studies have shown that apart from pressure overload and inherited forms of cardiomyopathies, oxidative stress can arise through mechanisms involving metabolic disturbances, mitochondrial dysfunction and inflammation amongst other dysregulated cellular processes, thereby revealing oxidative stress to be an extremely complex phenomenon that may explain as to why targeting of certain arms of oxidative stress alone may not be beneficial as patients with cardiac hypertrophy could present one of more of the above perturbations.

With the aim of understanding the complexities associated with oxidative stress, here we review the recent literature regarding oxidative stress-mediated cardiac hypertrophy and evaluate the mechanisms by which it is induced as well as discuss novel therapeutic approaches that may have the potential for attenuating cardiac hypertrophy and improving clinical outcomes.

\section{MEDIATORS OF OXIDATIVE STRESS ROS generating enzymes}

Nicotinamide adenine dinucleotide phosphate oxidase (NOX) proteins are reported to generate ROS in a highly regulated manner [14]. Several NOX isoforms have been identified and show tissue-specific distribution out of which NOX2 and NOX4 are the predominant isoforms expressed in the heart. Dysregulated expression of NOX proteins has been proposed to contribute to the development of cardiac hypertrophy and cardiac dysfunction. Consistently, elevated levels of NOX2 have been associated with worsening systolic function by a mechanism involving downregulation of PPAR $\alpha$ [15], an effect that could be reversed with PPARa activator fenofibrate or NOX2 inhibitor VAS2870. In the same study, transcriptomic analysis of PPARa ${ }^{-1-}$ mice subjected to pressure overload revealed alterations in oxidative stress and inflammatory pathways that overlapped with wild-type mice (also subjected to pressure overload), while NOX2 ${ }^{-/}$mice subjected to pressure overload exhibited minimal changes in these pathways. While this reinforces that dysregulated NOX2 expression is a critical inducer of oxidative stress and remodelling, its relationship with PPARa requires further investigation, although it can be speculated that downregulation of PPARa-target genes (e.g. carnitine palmitoyltransferase I and mediumchain acyl-CoA dehydrogenase) may lead to reductions in mitochondrial membrane potential, increased cellular ROS and calcium levels with accompanying perturbations in mitochondrial activity and ATP generation [16].

NOX4 is expressed primarily in the mitochondria of cardiomyocytes and initial studies have suggested that elevated NOX4 levels exert detrimental effects as its upregulation in response to hypertrophic stimuli and aging was found to induce oxidative stress, apoptosis and cardiac dysfunction. This was attributed in part to mitochondrial dysfunction due to ROS production and cysteine oxidation of mitochondrial proteins 
$[17,18]$. Angiotensin II (a hypertrophic stimulus) has also been shown to increase NOX4 levels which in turn induced nuclear export of HDAC4, a crucial suppressor of cardiac hypertrophy [19]. The dipeptidyl peptidase 4 inhibitor, teneligliptin, was able to attenuate cardiac hypertrophy and prevent increases in NOX4 mRNA, 4hydroxynonenal and HDAC4 export by augmenting GLP-1 levels, while the GLP-1 receptor antagonist, exendin-3, abrogated teneligliptin-mediated protection, revealing the existence of a GLP-1/NOX4/HDAC4 axis.

In contrast to these findings, NOX4 has been shown to mediate protection against pressure overload by preserving myocardial capillary density [20], but not volume overload, where it promoted the latter by increasing Akt phosphorylation (as a result of NOX4-mediated SRC kinase-dependent inactivation of protein phosphatase 2A) and modulation of downstream proteins RPS6 and 4E-BP1 [21]. Moreover, NOX41- mice exhibited less eccentric LV remodelling and reduced cardiomyocyte enlargement that was attributed to reduction in phosphorylated Akt levels. Conversely, cardiomyocyte-targeted NOX4 overexpressing hearts were found to preferentially oxidise fatty acids for energy production, thereby improving myocardial bioenergetics in the setting of pressure overload [22]. Finally, NOX4 has been found to be downregulated in the hearts of prenatally androgenised female offspring that exhibit cardiac hypertrophy in adulthood [23], highlighting the protective and diverse nature of this isoform.

Though NOX2 and NOX4 have been the main focus of oxidative stress, recent evidence suggest that other NOX proteins are also expressed in the heart and are mediators of cardiac hypertrophy. The calcium-regulated NOX5 isoform has been implicated in cardiac remodelling and contractile dysfunction that occurs in the setting of pressure overload [24]. In this study, overexpression of NOX5 was found to aggravate the hypertrophic response with transgenic NOX5 mice demonstrating greater magnitudes of hypertrophy, fibrosis and worsened contractile function. Treatment with either the ROS inhibitor $\mathrm{N}$-acetylcysteine (NAC) or the L-type calcium channel blocker diltiazem was shown to mitigate the adverse effects mediated by NOX5 overexpression, thereby placing NOX5 as a key intermediate between ROS and calcium signalling networks. In other studies, lysyl oxidase (LOX), a copperdependent amine oxidase that is reported to control matrix remodelling has also been found to aggravate the hypertrophic response, as mice overexpressing human LOX demonstrated heightened cardiac hypertrophy and cardiac dysfunction that was attributed to an enhanced fibrotic response as a result of excessive inflammation and ROS production which potentiated the activation of p38 MAPK while reducing AMPactivated protein kinase (AMPK) activation [25], suggesting new roles for LOX, apart from modulating collagen cross-linking.

In summary, based on several studies, dysregulated NOX expression has been found to be a key pathophysiological factor in the setting of cardiac hypertrophy (Figure 1). Further studies are needed to understand the interplay between the various NOX isoforms, their level of activation, and importantly, the downstream signalling pathways which they regulate. Future studies will need to take into consideration the differential responses of NOX proteins to varying types of hypertrophic stimuli 
(pressure overload vs angiotensin II) [26]. Until then, the use of non-isoform selective NOX inhibitors for disease management should be viewed with caution.

\section{Metabolic disturbances}

Recently, several studies have shown diabetes and obesity to play detrimental roles in the progression of cardiac hypertrophy, thereby warranting a deeper understanding of the mechanisms involved (Figure 2). For instance, diabetic rats have been found to develop cardiac hypertrophy and fibrosis with increased myocardial levels of NOX2 and NOX4 [27]. Moreover, mice subjected to high-fat diet (HFD) were found to exhibit elevated NOX2 levels with accompanying increases in cardiomyocyte size, oxidative stress and aberrant redox signalling as well as increased Akt and Erk1/2 phosphorylation [28]. In this study, oxidative stress could be suppressed when heart homogenates were treated with the superoxide scavenger, Tiron, the flavohaemoprotein inhibitor diphenyleneiodonium and the NOX2 assembly inhibitor NOX2 ds-tat. Moreover, the above abnormalities were abrogated in NOX2-KO hearts, highlighting NOX2 as a key mediator of obesity-induced cardiac hypertrophy. Although the effect of NOX2-KO on cardiac function was not investigated in this study, but in another study improved cardiac systolic function has been observed in $\mathrm{NOX}^{-/-}$mice in the setting of pressure overload [15]. It therefore appears that dysregulated NOX2 expression is a common determinant of both obesity- and pressure-induced cardiac hypertrophy. In other studies, oxidative stress induced by HFD was found to activate a BCL10/CARD9/p38 MAPK axis that could be supressed by zinc-mediated activation of metallothionein [29], revealing the existence of signalling intermediates between oxidative stress and conventional inducers of cardiac hypertrophy.

Considering the detrimental effects mediated by HFD, it is not unreasonable to speculate that lipid metabolism regulatory pathways may contribute to disease progression or prevention. For instance, knockdown of CTRP9, a gene involved in the regulation of lipid metabolism was found to aggravate cardiac hypertrophy, fibrosis, endoplasmic reticulum stress-initiated apoptosis and oxidative stress in the setting of obesity-induced cardiac hypertrophy, effects which were attributed to decreased AMPK phosphorylation and increased mTOR phosphorylation [30]. Moreover, in the setting of palmitate-induced lipotoxicity, treatment with CTRP9 protein was shown to increase LKB1 phosphorylation which in turn promoted its cytoplasmic localisation that is critical for AMPK activation, highlighting anti-lipotoxic properties likely mediated through a LKB1/AMPK axis. In other studies, palmitate treatment has been found to induce cristae remodelling, mitochondrial fission and ROS generation [31], where mechanistic studies linked these phenomena to decreased AMPK expression which in turn inhibits mitofilin interaction with both SAM50 and CHCHD3, two core components of the MICOS complex. Interestingly, the major neurotransmitter acetylcholine was shown to alleviate cardiomyocyte hypertrophy by improving cristae remodelling and mitochondrial function which was likely mediated through increased mitofilin expression and AMPK activation.

Lipid kinases such as PIKfyve are reported to be key regulators of cardiometabolic status and mitochondrial integrity as it has been found to induce 
mitochondrial ROS production and apoptosis [32]. Inhibition of PIKfyve through the use of a selective inhibitor STA was shown to suppress oxidative stress, apoptosis and mitochondrial damage which resulted in attenuated cardiac hypertrophy and improved cardiac function through a SIRT3-dependent pathway. Consistently, SIRT3 activation has been found to be protective against mitochondrial and energy metabolic dysfunction mediated by RIP140, a deleterious regulator of cardiac mitochondrial function and energy metabolic homeostasis [33]. Moreover, other lipid regulatory proteins like perilipin 5 (PLIN5) which is involved in the metabolism of lipid droplets and which is highly expressed in oxidative tissue such as in the heart have also been investigated. In the setting of pressure overload, mice deficient for PLIN5 were found to exhibit aggravated cardiac hypertrophy and dysfunction with accompanying reductions in cardiac lipid accumulation, increased mitochondrial biogenesis and fatty acid oxidation as well as heightened oxidative stress [34]. These observations support a role for PLIN5 in the protection of myocardial triglyceride stores, preservation of fatty acid oxidation, and prevention of oxidative stress.

Apart from excess fatty acids, hyperglycaemic conditions have also been shown to induce cardiomyocyte hypertrophy as incubation of H9c2 cells in high glucose concentrations resulted in increased cell size and increased cellular calcium and hypertrophic signals in addition to excess ROS production [35]. Interestingly, the PPARס agonist, GW0742, was shown to mitigate high glucose-induced cardiomyocyte hypertrophy by decreasing cellular calcium which in turn reduced ROS production and subsequent hypertrophic signalling. With regard to the mechanism, hyperglycaemic conditions were found to reduce thioredoxin 2 levels (a mitochondrial antioxidant that along with thioredoxin reductase 2 and peroxiredoxin 3 protects against oxidative stress) in both $\mathrm{H} 9 \mathrm{c} 2$ cells and in myocardium of diabetic rats [36], highlighting a direct effect of hyperglycaemia on redox balance.

Although metabolic disturbances have been found to be associated with adverse cardiac remodelling it is unclear as to whether the accompanying oxidative stress is the primary cause of disease manifestation or secondary to other pathophysiological events, although recent evidence suggests the former. When cardiac metabolism, function and structure were evaluated in a time course study in spontaneously hypertensive rats, chronic pressure overload was found to induce increases in myocardial glucose uptake and oxidation as well as elevate oxidative stress and inflammation and induce metabolite abnormalities that coincide with, or precede cardiac dysfunction while LVH developed later on [37]. While it remains to be determined as to how these early metabolic changes give rise to $L V H$, the activation of mTOR has been proposed as a contributing factor. Similarly, profound myocardial metabolic changes have been found to occur prior to the progression of LVH and cardiac dysfunction in spontaneously hypertensive rats [38].

In light of these observations, the modulation of metabolism has gained much interest as a potential therapeutic approach for reversing adverse events associated with cardiac hypertrophy. For instance, the glucose lowering agent metformin was shown to mitigate early myocardial metabolic changes by normalising glucose uptake rates and reducing circulating free fatty acids (fatty acyl carnitines) in addition to 
exerting positive effects on LV mass and LV wall thickness which resulted in improved cardiac function [38]. The beneficial effects of metformin have been attributed to the normalising of AMPK and mTOR signalling pathways as well as enhanced fatty acid oxidation and reductions in oxidative stress. Moreover, in a clinical study that evaluated the ability of metformin to reduce LVH in patients with coronary artery disease (with insulin resistance but without type 2 diabetes), metformin treatment was reported to remarkably reduce LV mass index, LV mass, systolic blood pressure, body weight and oxidative stress [39].

In other studies, the rate-limiting enzyme of fatty acid $\beta$-oxidation, short-chain acyl-CoA dehydrogenase (SCAD) is reported to negatively regulate cardiac hypertrophy and fibrosis [40]. Consistently, by increasing expression and enzyme activity of SCAD, flavin adenine dinucleotide treatment was shown to inhibit cardiomyocyte hypertrophy and cardiac fibroblast proliferation [41] that was attributed to increased fatty acid oxidation and decreased ROS production. Similarly, allylmethylsulfide, a novel sulphur metabolite of garlic was shown to attenuate cardiac hypertrophy and fibrosis by reducing oxidative stress (via reducing lipid peroxidation and improving exogenous antioxidant activity) and apoptosis in addition to stabilising extra cellular matrix components [42], likely mediated through $\mathrm{Na}^{+} / \mathrm{K}^{+}-\mathrm{ATPase}$ [43].

Finally, caloric restriction itself has been found to protect against adverse cardiac remodelling in diabetic and obese mouse models by normalising iron homeostasis which subsequently resulted in decreased oxidative stress and inflammation [44]. Moreover, caloric restriction was shown to attenuate cardiac hypertrophy by improving intracellular redox balance through a mechanism involving mitoKATP activation [45]. Although the precise anti-hypertrophic mechanism mediated by caloric restriction is undetermined, it could be speculated that caloric restriction reduces oxidative stress and increases cellular antioxidant capacity. Conversely, while chronic exercise was shown to attenuate cardiac hypertrophy and fibrosis, it was unable to restore the nitroso-redox imbalance imposed by diabetes [27].

In summary, the above findings highlight as to how metabolic disturbances could induce a hypertrophic response through mechanisms involving oxidative stress. Further studies are needed to assess as to whether oxidative stress impacts metabolic regulators with subsequent perturbations in substrate preference and bioenergetic impairment which is known to occur in the setting of cardiac hypertrophy.

\section{Mitochondrial dysfunction}

Mitochondria are the powerhouse of the cell and apart from generating ATP, these organelles play an important role in maintaining cellular redox balance. As a consequence of ATP production, the electron transport chain complexes are a major source of ROS generation and while this is tightly regulated by antioxidant defence systems, an imbalance between the production of free radicals and the ability to detoxify these species could result in oxidative stress (Figure 3). Unsurprisingly, disturbances in mitochondrial or antioxidant proteins have been associated with cardiac hypertrophy. In patients with $\mathrm{HCM}$, mitochondrial complex I has been found to be upregulated with elevated ATP levels and increased antioxidant enzymes [46], 
suggesting hyperactivity of complex I which was proposed to contribute to elevated ROS levels. ROS can also effect ATP levels as evidenced by a study which compared differential status of energy metabolism between myocardial infarction and pathological hypertrophy, where ATP levels were found to be significantly impaired in the former, while being preserved in the latter [47]. This phenomenon was attributed to a PGC1 $\alpha / E R R a$ axis that remained active during myocardial infarction, but not during cardiac hypertrophy. Mechanistic studies indicated ROS to negatively regulate NF-KB by oxidation of cysteine residues on its DNA binding element which allowed for the preservation of the PGC1 1 /ERRa axis during myocardial infarction which in turn inhibited pyruvate dehydrogenase via activation of PDK4. Considering that ROS levels are also elevated during cardiac hypertrophy, further work is needed to determine why NF-KB was not deactivated (with subsequent activation of PGC1 1 /ERRa signalling) in this setting.

Abnormal mitochondrial ion homeostasis has also been associated with cardiac hypertrophy. For instance, high-salt diet was found to increase cardiac mitochondrial TRPC3 expression which resulted in enhanced mitochondrial calcium uptake and ROS production [39]. This was accompanied by cardiac hypertrophy and mitochondrial dysfunction as evidenced by decreased ATP production and mitochondrial complex I and II enzyme activity. Moreover, as TRPC3 depletion was shown to attenuate cardiac hypertrophy and improve mitochondrial function, the importance of regulated ion uptake was highlighted in this study. Mitochondrial dysfunction as a result of iron overload has also been investigated in the setting of apelin-13-induced cardiac hypertrophy, where apelin-13 (an endogenous ligand for the $G$ protein-coupled apelin receptor) was found to promote total cellular and mitochondrial iron production with accompanying increases in mitochondrial ROS [48]. Mechanistically, this phenomenon was attributed to increases in mitochondrial iron transporting protein SFXN1 and a cargo receptor for ferritinophagy, NCOA4 suggesting that the endogenous ferritinophagy pathway may be a critical molecular switch in the development of cardiac hypertrophy.

Perturbations in mtDNA has been shown to have severe consequences as accumulation of mtDNA damage with age has been found to induce cardiac hypertrophy and dilation with accompanying impairments in systolic and diastolic function partly mediate by mitochondrial oxidative stress [49]. Endonuclease $G$ (ENDOG) which encodes for a mitochondrial nuclease has been identified as a determinant of cardiac hypertrophy, as its deficiency was found to induce ROS production and cell enlargement by altering the Akt/GSK3 $\beta$ and class-II HDAC signalling cascades [50]. Paradoxically, no changes were detected in the expression and activity of respiratory chain complexes nor in ATP content despite reductions in mtDNA as a consequence of ENDOG deficiency. Interestingly, treatment with humanin, a micropeptide coded by mtDNA was able to restore ROS levels and normalise cell size in ENDOG-deficient cardiomyocytes suggesting interplay between mtDNA content and cell growth.

The role of MTG1 that regulates mitochondrial ribosome assembly and translation has been investigated in cardiac hypertrophy [51]. While MTG1 
overexpression was found to confer protection, MTG1 deficiency significantly exacerbated the hypertrophic phenotype. Mechanistic studies revealed MTG1 to preserve mitochondrial respiratory chain complex activity which in turn reduced ROS production in addition to downregulation of TAK1, p38 MAPK and Jnk1/2 stress signalling pathways in the setting of pressure overload. Considering that MTG1 was found to be elevated in the hearts of patients with dilated cardiomyopathy and in mice subjected to pressure overload, further investigation is needed to determine as to why there was lack of protection in these settings. In other studies, TIM50 was found to be down-regulated in hypertrophied hearts and was associated with an aggravated hypertrophic response characterised by increased cardiomyocyte size and fibrosis [52]. While TIM50 deficiency was found to signal through an ASK1/Jnk/p38 MAPK axis, the ability for the antioxidant NAC to reverse the aggravated phenotype would suggest TIM50 deficiency to be associated with oxidative stress. This was supported by findings that revealed TIM50 to regulate ROS generation and activities of superoxide dismutase and catalase as well as complexes I, II and IV of the electron transport chain. Finally, uncoupling between gap junction proteins and mitochondria function has also been observed in cardiac hypertrophy as evidenced by the decreased expression of connexin 43 and GJA1-20 in spontaneously hypertensive rats [53]. Although overexpression of GJA1-20 was shown to increase mitochondrial membrane potential and oxygen consumption rates while reducing cell size and attenuating ROS levels, these improvements observed on mitochondria function are likely to be secondary to the chaperoning of connexin 43 , which was shown to be the primary function of GJA1-20.

In an attempt to protect mitochondria from oxidative damage, attention has been focussed on the development of antioxidants that can penetrate the mitochondria. In this regard, the mitochondria-targeted antioxidant Mito $Q$ has been shown to protect against the development of cardiac hypertrophy likely mediated by improved endothelial function as a result of increased nitric oxide bioavailability [54]. Similarly, the combination of MitoQ10 and low-dose losartan has been shown to provide additive protection by attenuating the development of hypertension and reducing cardiac hypertrophy [55]. Alternatively, targeting of mitochondrial dynamics could also be considered as a potential therapeutic strategy as administration of Drp1 inhibitor mdivi-1 in the setting of hypertension was shown to attenuate LVH and reduce fibrosis in addition to decreasing ROS production which subsequently supressed calcineurin and CaMKII expression [56].

In summary, these studies demonstrate that in addition to ATP production, mitochondria play a central role in preventing ROS accumulation. The close association between oxidative stress and perturbations in mtDNA, ion homeostasis, ribosome assembly, protein translocation as well as dynamics suggests mitochondrial dysfunction to be a critical mediator of oxidative stress-induced cardiac hypertrophy, thereby warranting a deeper understanding of the pathways involved.

\section{Inflammation}


Inflammation is a complex biological response to cellular or tissue damage and is associated with myocardial fibrosis, diastolic dysfunction and cardiac hypertrophy [57]. Whether inflammation induces oxidative stress in the setting of cardiac hypertrophy is less understood. Toll-like receptor 4 (TLR4) signalling is a critical link between oxidative stress, inflammation and cardiovascular disease [58]. In support, in a rat model of pre-term birth that was exposed to high levels of oxygen, an increase in TLR4 signalling was found to induce ROS production, inflammation and $\mathrm{CD}^{2} 8^{+}$macrophage infiltration which resulted in cardiac hypertrophy, fibrosis and cardiac dysfunction, all of which were prevented upon treatment with a TLR4 antagonist [59]. Moreover, TLR4 expression has also been found to be markedly increased with accompanying mitochondrial dysfunction and disturbed cellular antioxidant flux during isoproterenolinduced cardiac hypertrophy [60]. In this study, while these perturbations were shown to be prevented in the presence of a TLR4 receptor inhibitor RS-LPS, treatment with the TLR4 agonist LPS aggravated oxidative stress and accelerated disease progression, thereby highlighting inflammation as a crucial mediator of cardiac hypertrophy through induction of oxidative stress.

Nrf2 is a transcription factor that is reported to play a critical role in preventing oxidative stress and cardiac hypertrophy as its deficiency has been found to aggravate cardiac hypertrophy, fibrosis, oxidative stress, inflammation and cardiac dysfunction through an IL-6/STAT3 axis [61]. In an attempt to preserve Nrf2 signalling, overexpression of the antioxidant peroxiredoxin 1 exerted protection through a Nrf2/HO-1 axis which led to decreased inflammation and oxidative stress [62]. Interestingly, though peroxiredoxin 1 was inherently upregulated in the setting of pressure overload, these levels were not sufficient to prevent disease onset, allowing for the speculation that other signalling intermediates could be present in this cascade. Moreover, inflammation has also been found to signal through regulators of DNA as the transcriptional and epigenetic regulator bromodomain-containing protein 4 (BRD4) was found to be upregulated in cardiomyocytes following exposure to angiotensin II. This was attributed to increased ROS generation as both NAC treatment and BRD4 suppression were able to limit cell enlargement, repress TGF $\beta 1 / S M A D$ signalling pathways as well as alleviate inflammation and oxidative stress through inhibition of NF-KB signalling and improved Nrf2/HO-1 signalling, respectively [63]. In the same study, BRD4 deficient mice were reported to exhibit protection against pressure overload as evidenced by reductions in cardiac hypertrophy, fibrosis, inflammation, oxidative stress and cardiac dysfunction.

Adipokines are inflammatory mediators that are also associated with cardiac hypertrophy as cardiomyocyte-specific adipokine, CTRP3, has been found to be upregulated in murine hypertrophic hearts and failing human hearts [64]. The increase in CTRP3 was found to be induced by ROS during the hypertrophic response and while overexpression of CTRP3 worsened cardiac hypertrophy and function, CTRP3 deficiency alleviated the hypertrophic phenotype. Mechanistic studies revealed CTRP3 to signal through a TAK1/Jnk axis that was initiated by PKA. Moreover, secretory proteins have also been investigated in an attempt to identify their role in cardiac hypertrophy. FNDC5 plays an important role during muscle contraction in that 
it undergoes cleavage to release the myokine irisin into the bloodstream which in turn promotes mitochondrial biogenesis and increases metabolic rate in cardiomyocytes [58]. In support, FNDC5 deficiency in the setting of HFD aggravated cardiac hypertrophy and increased inflammatory cytokine expression and oxidative stress that was attributed to activation of the JAK2/STAT3 pathway [65]. Although it remains to be determined as to how FNDC5 modulates the JAK2/STAT3 signalling axis, this study highlights the importance of myokines in maintaining cardiac homeostasis. Finally, treatment with FGF21 (a hepatokine) was found to attenuate cardiac hypertrophy, fibrosis, apoptosis and cardiac dysfunction that was likely mediated by an increase in the deacetylase activity of SIRT1, which promoted its interaction with LKB1 and FOXO1 [66]. While deacetylation of LKB1 resulted in activation of AMPK, deacetylation of FOXO1 led to altered transcriptional activity which resulted in increased expression of catalase and MnSOD and decreased expression of proapoptotic protein BIM. These findings support a critical role of adipokines, myokines and hepatokines in regulating inflammatory responses in the setting of cardiac hypertrophy.

In other studies, molecules associated with the innate immune system like C1QTNFs have been reported to display differential effects on metabolic homeostasis which could influence the cardiovascular system [67]. Consistently, C1QTNF1 has been found to be elevated in response to ROS whereas its deficiency led to accelerated cardiac hypertrophy and remodelling with increased inflammation and oxidative stress and worsened cardiac function [68]. Introduction of the recombinant human globular domain of C1QTNF1 mitigated the hypertrophic response through AMPKa activation which in turn supressed mTOR and P70S6K phosphorylation with mechanistic studies revealing the existence of a cAMP/PKA/LKB1 axis that was a critical prerequisite for C1QTNF1-mediated AMPKa activation.

Other cell types have also been reported to promote an inflammatory response in the setting of cardiac hypertrophy. For instance, endothelial cells have been found to secrete endothelial microparticles (EMP) containing mitochondria and if these mitochondria were dysfunctional (excessive ROS production), EMPs were then able to induce expression of proinflammatory mediators in endothelial cells which in turn increased monocyte adhesion [69]. Mechanistically, MK2 was found to regulate EMP generation during inflammation by increasing E-selectin expression and cytoskeletal rearrangement through ROCK2. In the same study, MK2 inhibition was shown to attenuate cardiac hypertrophy and fibrosis as well as improve cardiac function, highlighting as to how paracrine mediators such as EMP could influence disease pathophysiology. Consistently, when investigating the relationship between plasma xanthine oxidase (a product of endothelial cells) and LVH in patients with resistant hypertension (RHTN), a two-fold increase in xanthine oxidase was observed in RHTN patients as compared to normotensive persons which was positively correlated with LV mass, LV diastolic function and 24-hour urinary sodium [70], highlighting an association between endothelium-derived oxidative stress and excess dietary salt in LVH pathophysiology. 
In summary, based on these observations it would seem that not only does inflammation induce oxidative stress but the reverse is also true, suggestive of a feedback loop between these two physiological processes. Moreover, though inflammation is a well-documented occurrence in the setting of cardiac hypertrophy, not all mediators of inflammation are detrimental and hence, more studies are needed to delineate the relationship between pro- and anti-inflammatory pathways with regard to oxidative stress, as suppression of the wrong cascade could have deleterious outcomes (Figure 4).

\section{Autophagy}

Cardiomyocyte growth is a key pathophysiological feature of cardiac hypertrophy, necessitating the need for understanding its regulation. In support, CDC20, an anaphase-promoting complex activator essential for cell division has been found to be up-regulated in the hypertrophied heart [71]. While knockdown of CDC20 attenuated cardiac hypertrophy, ectopic expression of CDC20 aggravated the hypertrophic response by directly targeting and promoting ubiquitination and degradation of the critical autophagy regulator LC3, thereby impairing autophagy and promoting cardiac hypertrophy. This study highlights a critical link between cell division proteins, the autophagy machinery and the hypertrophic response.

Apart from acquired LVH and HCM, Fabry disease is a rare genetic disorder caused by defects in the alpha-galactosidase (GLA) enzyme which results in globotriaosylceramide (Gb3) accumulation. Although considered a multi-system disorder, LVH is a key pathophysiological feature being reported in up to $50 \%$ of males and one-third of females [72]. Consistently, when the GLA gene was knocked out in human embryonic stem cells (hESCs), the differentiated cardiomyocytes were found to exhibit cellular hypertrophy with accompanying accumulation of $\mathrm{Gb} 3$ and decreases in the Rab GTPases involved in exocytotic vesicle release [73]. This resulted in impaired autophagy flux and protein turnover which in turn induced ROS production and apoptosis, however, further work is needed to determine the interplay between exosome biogenesis and autophagy.

Since impaired autophagy is known to destabilise proteostasis and elevate intracellular oxidative stress during cardiac remodelling, targeting this pathway has been proposed as a viable strategy for mitigating the hypertrophic phenotype. In support, oridonin, the major active ingredient of the traditional Chinese medicinal herb Rabdosia rubescens was shown to attenuate cardiac hypertrophy and preserve heart function in the setting of pressure overload [74]. While the beneficial effects of oridonin were attributed to increased antioxidant activities and suppressed oxidative injury (which were a result of intracellular protein accumulation), mechanistically this was linked to enhanced myocardial autophagy mediated by increased levels of cytosolic p21 which reduced Akt but increased AMPK phosphorylation. While enhancing autophagy appears to be an attractive strategy, caution is advised as excessive autophagy has been reported to be maladaptive. In support, hypertrophic stimuli were found to induce autophagy through a mechanism involving NOX2. Interestingly, stachydrine, a major constituent of Leonurus heterophyllus Sweet was shown to inhibit 
NOX2 activity which led to reductions in ROS and subsequent attenuation of cardiac hypertrophy and cardiac dysfunction as well as decreased excessive autophagy [75]. Moreover, apelin-13 was found to promote cellular hypertrophy through an endoplasmic reticulum stress-autophagy pathway as evidenced by increases in ROS production, NOX4 expression, endoplasmic reticulum stress markers (BiP and CHOP) and autophagy markers (LC3-II/I and beclin-1) [76], suggesting interplay between ROS, endoplasmic reticulum stress and autophagy.

In summary, while studies suggest autophagy to be involved in both promoting and preventing cardiac hypertrophy (Figure 5), the occurrence of reticulophagy (a selective form of autophagy of the endoplasmic reticulum) was found to occur in the setting of apelin-13 induced cardiomyocyte hypertrophy and further investigation is needed to determine as to how such specificity arises [76].

\section{Protein quality control}

Protein quality control is a cellular phenomenon through which aberrant proteins are eliminated and hence, maintaining functional protein homeostasis is important as disruptions in these pathways could have detrimental effects on the cell. In the setting of cardiac hypertrophy TRAF6, a ubiquitin E3 ligase was found to be elevated in human and murine hypertrophied hearts [77]. Mechanistically, TRAF6 was found to be regulated by ROS generated during the hypertrophic progression which triggered its auto-ubiquitination that led to the formation of a TRAF6-TAK1 interaction that was indispensable for cardiac remodelling. Moreover, REGy, a member of the $11 \mathrm{~S}$ proteasome activator is reported to bind and activate the $20 \mathrm{~S}$ proteasome to promote the degradation of several proteins [78]. In the setting of pressure overload, REGy was found to be up-regulated and associated with increased decay of PP2Aca, which resulted in increased phosphorylation and nuclear export of FOXO3a which subsequently led to a decline in MnSOD and ROS accumulation [79]. In the same study, ectopic expression of PP2Aca or MnSOD was shown to mitigate REGYmediated ROS accumulation and treatment with a MnSOD mimetic, MnTBAP was found to prevent ROS accumulation and cardiac hypertrophy highlighting REGy/PP2Aca-FOXO3a/MnSOD as an important signalling pathway (Figure 5).

In other studies, continuous overexpression of the small heat shock protein Hsp22 in mice has been found to increase ROS production which in turn induced cardiac hypertrophy, senescence (as evidenced by increased p16 and p19 levels, percentage of $\beta$-galactosidase positive cells and telomerase activity) and a reduction in lifespan [80]. In this study, the 3 major cellular sources of ROS (NADPH oxidase, xanthine oxidase and complex I) were found to have elevated activity that could be abolished with the antioxidant tempol which in turn attenuated cardiac hypertrophy, prevented senescence and extended the life span likely through modulation of Akt phosphorylation. Although it remains to be determined as to how Hsp22 enhanced the activity of ROS producing proteins, it can be speculated that this could be due to their stabilisation, as Hsp22 is reported to have chaperoning properties [81].

In summary, studies have shown dysregulated protein quality control to be a mediator of oxidative stress and cardiac hypertrophy through heightened proteasomal 
activity that results in the perturbation of protective pathways and in the probable stabilisation of ROS producing enzymes.

\section{miRNAs}

miRNAs are small non-coding RNA molecules that are involved in the posttranscriptional regulation of genes. Several miRNAs have been found to commonly contribute to cardiac hypertrophy. For instance, miR-29 has been implicated in HCM where its silencing has been associated with increased cardiac fibrosis [82]. With regard to its regulation, endothelin-1 has been proposed as an upstream regulator of miR-29a as treatment of cardiomyocytes with endothelin-1 was found to increase ROS and suppress miR-29 with corresponding increase in TGF $\beta$ expression, which in turn stimulated collagen expression in fibroblasts. In the same study, it was found that suppression of miR-29 was only observed in the R92W-TnT mouse model and not in the R403Q-MyHC model nor in human myomectomy samples, where the latter surprisingly demonstrated activation of anti-hypertrophic/anti-fibrotic signalling pathways. It can be speculated that while differences between the two models may be due to their differential redox status, the activation of anti-hypertrophic/anti-fibrotic signalling pathways in human samples may be a result of compensatory mechanisms.

Conversely, the levels of miR-200c has been found to be increased during cardiac hypertrophy and has been shown to be a direct target of MLCK and hence, an increase in miR-200c induced a decrease in phosphorylated MLC2 [83]. Interestingly, down-regulation of miR-200c using a specific inhibitor was shown to attenuate cardiomyocyte hypertrophy and reduce apoptosis as well as ROS accumulation, effects that were lost upon MLCK inhibition, thereby highlighting a direct link between miRNAs, sarcomere function and ROS.

In summary, though dysregulation of miRNAs has been found to occur during cardiac hypertrophy (Figure 5) it is unclear as to whether they mediate disease pathology through mechanisms involving oxidative stress, although miRNAs have been found to regulate mitochondrial proteins in response to hypertrophic stimuli, as miR-28 was found to target the 3 untranslated region of VDAC1 [16], while miR-106a was found to target Mfn2 resulting in cristae defects, depolarization of mitochondrial membrane and increased ROS production [84], allowing for the speculate of an association between miRNAs, mitochondrial protein and oxidative stress.

\section{Ion channels}

Cardiac ion channel complexes form the basis for excitation contraction coupling including calcium-induced calcium release and mechanical contraction and while ion channel dysregulation has been reported in cardiac hypertrophy [85], whether this is also associated with oxidative stress is less clear. In spontaneously hypertensive rats, the existence of an EGFR/NHE1 axis has been proposed as EGFR depletion led to reduction in NHE1 activity with accompanying attenuation of cardiac hypertrophy and decreased fractional shortening [86]. Although this signalling cascade requires further investigation, it can be speculated that NHE1 activation is ROS-dependent as EGFR depletion resulted in reduced NHE1 activity, albeit with no change in protein levels but 
with accompanying reductions in ROS and lipid peroxidation. In other studies, the expression and activity of NKA was found to be decreased in rodent cardiomyocytes exposed to angiotensin II [87]. Interestingly, treatment with an antibody against the $4^{\text {th }}$ extracellular region of NKA (DR-Ab) was shown to be protective as this led to stabilisation of NKA on the plasma membrane and restored its activity with accompanying reductions in ROS through NOX inhibition. Although mechanistic studies linked these beneficial effects of DR-Ab to the activation of an AMPK/SIRT3/PPARY axis, further studies are needed to validate the relationship between ion channel expression/activity and oxidative stress (Figure 6).

Recently, enhancement of the plasma membrane small conductance $\mathrm{Ca}^{2+}$ activated $\mathrm{K}^{+}$channels in hypertrophied hearts were shown to mediate protection against arrythmia by attenuating mitochondrial ROS and subsequently decreasing oxidation of reactive cysteine residues on ryanodine [88]. An association between impaired calcium handling and increased ROS production has also been observed in a hESC model of inherited hypertrophic cardiomyopathy caused by mutations in the muscle LIM protein (MLP), a key regulator of straited muscle function [89]. MLP deficiency was found to induce a hypertrophic phenotype in hESC-derived cardiomyocytes with accompanying mitochondrial damage, increased ROS production and impaired calcium handling. Interestingly, the calcium channel blocker verapamil was shown to prevent the development of $\mathrm{HCM}$ suggesting elevated intracellular calcium concentration to be a central mechanism for MPL-deficiency induced cardiac hypertrophy, although assessment of verapamil in reducing ROS levels was not investigated.

In summary, oxidative stress has been found to have a profound effect on cardiac ion channels. Considering the criticality of ion channels in regulating excitation contraction coupling, an extensive understanding of oxidative stress-induced modifications could help to identify potential therapies that could prevent incidences of arrythmia, which is a common occurrence in patients with cardiac hypertrophy.

\section{THERAPEUTIC STRATEGIES FOR ATTENUATING OXIDATIVE STRESS Antioxidants}

Considering that oxidative stress is a result of impaired antioxidant activities, several studies have focussed on augmenting these signalling cascades through direct or indirect intervention. In this regard, several naturally occurring compounds have been shown to alleviate the hypertrophic phenotype through suppression of oxidative stress. For instance, the pulp powder extracted from Musa balbisiana (banana) [90], the lowbush blueberry extract (Vaccinium angustifolium) [91], the aqueous extract of the Terminalia arjuna bark [92] and pomegranate juice [93] have been associated with reduction in heart weight, fibrosis, inflammation and oxidative stress. In addition to supressing cardiac hypertrophy, these compounds have been reported to exert beneficial effects across several pathologies suggestive of pleiotropic function. Moreover, while the proposed mode of action of these compounds is through suppression of oxidative stress, likely mediated by several phenolic antioxidants, the exact composition and the signalling pathways through which they signal are less 
defined, although quercetin, kaempferol, boeravinone B and caffeic acid have been identified in the ethanolic extract of Boerhavia diffusa [94].

Signalling cascades that regulate oxidative stress and/or antioxidant activity have however been identified in other studies. Nobiletin, a polymethoxy flavonoid was shown to attenuate cardiac hypertrophy by blunting the increased expression of NOX2 and NOX4 as well as alleviating endoplasmic reticulum stress and reducing cardiomyocyte apoptosis in the setting of pressure-overload [95]. In other studies, dihydromyricetin, a flavonoid in vine tea, has been reported to exert protection by reducing $\mathrm{ROS}$ and lipid peroxidation while increasing antioxidant expression and activity which was attributed to enhanced SIRT3 signalling [96]. However, not all members of the SIRT family are protective, and caution should be observed when targeting these proteins as SIRT4 overexpressing mice were found to exhibit aggravated hypertrophy, fibrosis and cardiac dysfunction in response to angiotensin II. This was attributed to less binding of MnSOD to SIRT3 which resulted in increased MnSOD acetylation and reduced activity, thereby inducing ROS accumulation [97]. Importantly, a mimetic of superoxide dismutase, manganese 5, 10, 15, 20-tetrakis-(4benzoic acid) porphyrin was shown to exert beneficial effects through inhibition of ROS.

While these compounds exert protection by augmenting antioxidant pathways and suppressing oxidative stress, their pleiotropic nature has been found to influence off-target signalling, albeit with beneficial effects. The natural flavonoid, delphinidin for instance has been shown to attenuate hypertrophic growth, reduce fibrosis and improve cardiac function which was attributed to its ability to reduce ROS accumulation by activating AMPK with subsequent inhibition of Rac1 (NOX activator) activity and p47 phox (a NOX subunit) expression [98]. Interestingly, delphinidin also abrogated oxidative stress-mediated increases in Erk1/2, p38 MAPK and Jnk1/2 phosphorylation, suggesting modulation of an AMPK/NOX/MAPK axis. Similarly, fisetin, a small molecular flavonoid was found to attenuate cardiac hypertrophy and improve cardiac function by decreasing ROS levels and up-regulating antioxidant genes including catalase, superoxide dismutase and HO-1 in addition to supressing pro-hypertrophic signalling pathways such as MAPK and mTOR [99]. In other studies, astragaloside IV, a bioactive saponin isolated from the dried plant roots of the genus Astragalus, has been shown to alleviate the hypertrophic phenotype by increasing Nrf2 and HO-1 levels and supressing ROS [100], astragaloside IV was also found to function through elevating SIKE (a negative regulator of the interferon pathway) which in turn supressed the TBK1/PI3K/Akt axis [101]. Moreover, rutaecarpine an alkaloid isolated from Evodia rutaecarpa, the most popular and multi-purpose herb traditionally used in China for treatment of several symptoms, was shown to attenuate cardiac hypertrophy through suppression of both a NOX4/ROS/ADAM17 axis and the prohypertrophic Erk1/2 pathway [102]. Additionally, ginsenoside $\mathrm{Rd}$, one of the main active ingredients in Panax ginseng, was also reported to improve cardiac function and attenuate cardiomyocyte hypertrophy, fibrosis, inflammation and oxidative stress with accompanying reductions in Akt, calcineurin A, Erk1/2 and TGF $\beta 1$ protein levels [103], thereby associating these compounds with anti-hypertrophic, anti-fibrotic, anti- 
inflammatory and antioxidant properties. Finally, antioxidants may also be considered for preventing remodelling of the $\mathrm{RV}$ as a result of pulmonary hypertension as phytophenol pterostilbene together with hydroxypropyl- $\beta$-cyclodextrin were shown to reduce production of NOX-dependent superoxide anions and oxidative stress and preserve systolic function [104].

Apart from modulating pro-hypertrophic signalling pathways, antioxidants have also been found to improve mitochondrial function. In addition to preventing accumulation of ROS and supressing ROS-dependent pro-hypertrophic MAPK and Akt signalling pathways, lycopene, a carotenoid antioxidant, was reported to prevent opening of the mitochondrial permeability transition pore and improve mitochondrial function, although its main mode of action was likely mediated through restoration of antioxidant response element activity and subsequent activation of antioxidant genes [105]. In other studies, isosteviol sodium which is a derivative of stevioside, a constituent of Stevia rebaudiana, which is commonly used as a noncaloric sugar substitute, has been reported to supress the hypertrophic response in addition to restoring mitochondrial membrane potential and decreasing fission proteins (Fis1 and Drp1) [106] with accompanying decreases in ROS and elevated antioxidant levels (thioredoxin 1 and peroxiredoxin 2). Finally, the polyphenolic compound resveratrol was found to maintain normal LV volumes and preserve systolic function in the setting of cardiolipin deficiency-induced cardiac hypertrophy [107], which was attributed to improvements in mitochondrial respiration as well as decreased ROS levels and oxidative damage to the myocardium.

Based on these studies, it is clearly evident from several models that antioxidants do exert protection by negatively impacting several pathways that regulate hypertrophic growth, fibrosis, inflammation and oxidative stress to attenuate the disease phenotype while improving cardiac function. However, in a double-blind, randomized, single-centre pilot study involving $\mathrm{HCM}$ patients, high-dose NAC treatment for 12 months was found to have small effects on indices of cardiac hypertrophy and fibrosis when compared to placebo group [108]. While these findings were unexpected, given that NAC had previously been shown to exert protection in several models of cardiac hypertrophy $[109,110]$, the disparate observations between animal and human studies could be attributed to the genetic heterogeneity of patients with $\mathrm{HCM}$ as well as differential redox status.

In summary, while antioxidants have been shown to be protective in several animal models of cardiac hypertrophy, understanding their composition and pleiotropic functions is a critical prerequisite, as administration of such compounds in the clinical setting could result in inconsistent outcomes that could be attributed to disease aetiology and patient heterogeneity (see Table 1 for summary).

\section{Selenoproteins}

The endoplasmic reticulum-resident selenoproteins are rapidly emerging as cardioprotective agents, as several studies have linked their dysfunction with susceptibility to oxidative stress and cardiovascular disease [111]. The involvement of selenoproteins in improving cardiac function in the setting of cardiac hypertrophy is 
less studied, however, recent studies have associated these proteins with favourable effects. In one study, pre-treatment of $\mathrm{H} 9 \mathrm{c} 2$ cells with $\mathrm{H}_{2} \mathrm{~S}$, was found to attenuate $\mathrm{H}_{2} \mathrm{O}_{2}$-induced cell death, oxidative stress and cell enlargement by activating the $\mathrm{SCLY} / \mathrm{H}_{2} \mathrm{Se}$ signalling cascade which in turn increased expression and activities of selenoproteins (glutathione peroxidase and thioredoxin reductase) [112]. As $\mathrm{H}_{2} \mathrm{~S}$ is endogenously produced by cystathionine gamma-lyase, mice deficient for this enzyme were found to display perturbed SCLY and selenoprotein P expression, though this effect on cardiac architecture or function was not investigated. While it would seem that both $\mathrm{H}_{2} \mathrm{~S}$ and $\mathrm{H}_{2} \mathrm{Se}$ may exert protection through specific activation of selenoproteins, the increase in Nrf2-target genes post-treatment may suggest improvements to a broader range of antioxidants. In support, when Dahl salt-sensitive rats were provided with high-salt diet, the myocardial $\mathrm{H}_{2} \mathrm{~S}$ pathway was found to be downregulated with elevated oxidative stress characterised by increased hydroxyl radicals, malondialdehyde and oxidised glutathione in addition to reduced total antioxidant capacity, carbon monoxide, catalase, glutathione, glutathione peroxidase and superoxide dismutase activities. Consistently, $\mathrm{H}_{2} \mathrm{~S}$ was able to normalise these levels with accompanying inhibition of cardiac hypertrophy [113]. In other studies, apart from attenuating the hypertrophic phenotype, administration of exogenous NaHS was found to exert broad protection in a SIRT3-dependent manner by increasing total antioxidant capacity and superoxide dismutase activity while reducing malondialdehyde and superoxide levels [114]. Interestingly, NaHS also had an effect on mitochondrial shaping proteins, as evidenced by increased Opa1 and decreased Drp1 levels. A similar observation was made in another study where NaHS treatment resulted in increased expression of mitochondrial fusion proteins (Opa1, Mfn1 and Mfn2) and decreased expression of fission proteins (Drp1 and Fis1) with accompanying improvements in mitochondrial function, permeability potential, ultrastructure and increased mitochondrial numbers [115]. These observations suggest $\mathrm{H}_{2} \mathrm{~S}$ to be pleiotropic as well, exerting a multitude of beneficial effect in the setting of cardiac hypertrophy, although enhancement of antioxidant capacity and suppression of oxidative stress may likely be its primary mode of action.

\section{Alternative therapies}

A cathelicidin-related antimicrobial peptide (CRAMP) has been shown to be cardioprotective in the setting of myocardial ischemia/reperfusion injury [116]. Similarly, CRAMP was found to protect against pressure overload by reducing the inflammatory response and oxidative stress [117]. Interestingly, CRAMP exhibited cardiomyocyte specificity as it was able to inhibit the hypertrophic response and oxidative stress in cardiomyocytes but not in in endothelial cells. Mechanistically, CRAMP was reported to exert its anti-hypertrophic effects by activating the IGFR1/PI3K/Akt pathway through direct binding to IGFR1 while its antioxidative effects were a result of TLR9/AMPKa activation. In other studies, an analogue of the $\alpha$ calcitonin gene-related peptide ( $\alpha$-CGRP) has been found to preserve heart function and prevent adverse cardiac remodelling and apoptosis in the setting of cardiac hypertrophy, highlighting the therapeutic potential of targeting the CGRP pathway 
[118]. Moreover, when considering therapeutic strategies for HCM, the sphingosine1-phosphate receptor modulator, fingolimod has been shown to improve diastolic function in the Tm-E180G mouse model of HCM [119], where mechanistic studies attributed these improvements to decreased S-glutathionylation of myosin-binding protein $\mathrm{C}$ and reduced oxidative stress via downregulation of NOX2. Though there was no change observed in the level of fibrosis, modulation of sphingolipid signalling may be a potential strategy for improving cardiac function in HCM patients.

Isolevuglandins (isoLGs) belong to a family of extremely reactive electrophiles and are generated by free radical-induced lipid oxidation and rearrangement of endoperoxide intermediates of the isoprostane pathway [120]. Considering the association between oxidative stress and cardiac hypertrophy, it is not surprising that an increase in isoLG protein adducts have been observed in cardiac tissue in the setting of pressure overload [121]. Interestingly, the IsoLG scavenger 2hydroxybenzylamine and its less reactive isomer 4-hydroxybenzylamine were shown to attenuate $\mathrm{LVH}$, reduce cardiac fibrosis and improve cardiac function, although the mechanisms remain unclear. In other studies, the analogues of imine stilbene (a compound that has previously been reported to exert cardioprotection and anti-aging via modulation of SIRT1) have also been found to alleviate the hypertrophic phenotype by suppressing several terminal stress kinases pathways [122]. It can be speculated that the imine stilbene analogue (compound $3 \mathrm{e}$ ) is able to maintain mitochondrial homeostasis through reduction in oxidative stress, as it was found to prevent $\mathrm{H}_{2} \mathrm{O}_{2}$ mediated apoptosis.

Cell therapy approaches can also be considered for mitigating cardiac hypertrophy as remote transplantation of human adipose-derived stem cells (hADSCs) into the right hamstring of spontaneously hypertensive rats was found to attenuate cardiac hypertrophy and fibrosis with accompanying reductions in myocardial ROS [123]. Interestingly, hADSCs pre-treated with n-butylidenephthalide (BP) were shown to reduce cardiac hypertrophy and ROS more profoundly than naïve hADSCs while on the molecular level this resulted in increased STAT3 phosphorylation with subsequent activation and nuclear translocation which likely induced elevation of myocardial interleukin 10 (anti-inflammatory) levels and M2 macrophage polarisation. This study demonstrates that transplantation of BP-treated hADSC could be considered as a strategy to reduce cardiac hypertrophy even at an established phase of hypertension. Similarly, gene therapy strategies could also be considered to counteract development of cardiac hypertrophy in the setting of pressure overload as selective high-density lipoprotein-raising human apolipoprotein A-I gene transfer was reported to reduce pathological cardiac remodelling, nitro-oxidative stress and apoptosis, while increasing myocardial capillary density and improving cardiac function [124].

Finally, treatment with intraperitoneal injection of hydrogen was shown to prevent cardiac hypertrophy and improve cardiac function through suppression of NOX expression and inhibition of mitochondrial membrane potential depression which in turn blocked ROS-sensitive Erk1/2, p38 MAPK and Jnk signalling pathways [125]. In other studies, the anion nitrite has also been associated with beneficial properties 
by improving antioxidant capacity in hypertensive rats, where a low nitrite dose was found to supress ROS production, prevent activation of the mTOR pathway and attenuate cardiac hypertrophy, but without affecting blood pressure [126]. Conversely, maternal exposure to nitrogen dioxide was found to induce cardiac hypertrophy in male offspring with accompanying elevation of cardiac injury markers and calcineurin activity that were attributed to the cardiac-specific transcription factor Csx/Nkx2.5 whose expression was regulated by a ROS/HIF1 $\alpha$ axis and DNA hypomethylation modification at its promoter [127].

In summary, apart from antioxidants and selenoproteins, alternative therapies that can attenuate cardiac hypertrophy and improve cardiac function through suppression of oxidative stress is an exciting prospect although the specificity of such compounds should be investigated further.

\section{Drug repurposing}

Drug repurposing is a strategy for identifying new uses of approved or investigational compounds that are outside the scope of the original medical indication. This strategy offers various advantages over developing an entirely new drug for a given indication. Statins are the most common cholesterol-lowering drugs that have been reported to improve morbidity and mortality in high risk patients with cardiovascular disease [128]. Statins are proposed to have pleiotropic effects and this has been observed in mice following infusion of angiotensin II where atorvastatin and pravastatin were shown to attenuate cardiomyocyte hypertrophy and fibrosis in wild-type mice but not in SmgGDS ${ }^{+/-}$mice, suggesting that statins function through small GTP-binding protein GDP dissociation stimulator which regulated Rac1 expression, Erk1/2 activity, Rhokinase activity and inflammatory cytokine secretion [129]. In another study that compared the effects of the sleep aid supplement, melatonin, in the setting of pathological and physiological cardiac hypertrophy, melatonin was found to attenuate oxidative stress and cardiac hypertrophy and improve cardiac function in the former but not in the latter [130]. Interestingly, melatonin was reported to signal through the retinoid-related orphan nuclear receptor- $\alpha(R O R \alpha)$ which was found to be otherwise decreased in human and murine pathological hypertrophic cardiomyocytes. Mechanistically, the beneficial effects of melatonin were attributed to transactivation of MnSOD by binding of RORa to its response element located at the MnSOD promoter region. Long-term treatment with melatonin has also been reported to attenuate cardiac hypertrophy, slow the deterioration of cardiac contractile function and improve survival rate by activating PGC1 $\beta$ and suppressing oxidative stress in the setting of pressure overload [94].

The effect of enalapril, a drug used to treat hypertension has also been investigated in the setting of ageing-related cardiac hypertrophy [131]. Interestingly, late-life enalapril treatment of aged rats resulted in attenuated cardiac hypertrophy and oxidative stress-related molecular damage with accompanying increase in mitochondrial antioxidant defences. Intriguingly, enalapril seemed to have a profound effect on the level of the mitochondria as evidenced by increased mitochondrial mass and biogenesis as well as up-regulated mitochondrial fusion signalling and autophagy. 
In other studies, celecoxib is a nonsteroidal anti-inflammatory drug that is used to treat arthritis amongst other conditions. Interestingly, in the setting of pressure overload, celecoxib was shown to attenuate cardiac hypertrophy and cardiac dysfunction by inhibiting apoptosis and inflammation via the MDM2/p53 pathway and the $\mathrm{Akt} / \mathrm{mTOR} / \mathrm{NF}-\mathrm{kB}$ pathways respectively, in addition to supressing oxidative stress via increases in Nrf2-mediated gene expression of multiple antioxidants [132]. Moreover, the traditional Indian Ayurveda medicine Yogendra Ras (YDR) has also been found to mitigate cardiac hypertrophy, which was attributed to the restoration of redox homeostasis as evidenced by suppression of biomarkers of oxidative stress and inflammation post-treatment [133]. Finally, cilostazol which is commonly used in the management of peripheral vascular disease [134] was shown to reduce nicotineinduced cardiomyocyte hypertrophy by enhancing cathepsin B activity which in turn resulted in improved autophagy flux and suppression of oxidative stress pathways [135].

In summary, while drug repurposing certainly seems an attractive prospect, caution is advised as certain compounds though beneficial for specific pathologies may exacerbate others. This has been observed with the PPARy agonist rosiglitazone (RSG) which is used to treat diabetes but has also been shown to stimulate cardiac hypertrophy and oxidative stress. Mechanistically, this has been linked to secretion of miR-200a from adipocytes which in turn was found to decrease tuberous sclerosis protein 1 and activate mTOR in cardiomyocytes thereby inducing a hypertrophic phenotype [136]. This study highlights the crosstalk between adipocytes and cardiomyocytes in regulating adverse cardiac remodelling.

\section{CONCLUSION}

Several studies have identified oxidative stress as a critical mediator of cardiac hypertrophy and consistently, targeting of oxidative stress has been shown to alleviate the hypertrophic response. Though initially thought to solely arise from pathways regulated by NADPH oxidase, xanthine oxidase and the mitochondrial electron transport chain, recent evidence has linked oxidative stress to metabolic disturbances, mitochondrial dysfunction, inflammation, impaired/overactive autophagy, dysregulated protein quality control and miRNA expression as well as impaired ion channel activity. Paradoxically, targeting of oxidative stress through the use of antioxidants in the clinical setting has yielded inconsistent results and diverse speculations have been made with regard to these unexpected findings including antioxidants being unable to reach the target cells in addition to not all ROS being detrimental and hence, targeting of ROS could induce a harmful reduced state. Here, we highlight that oxidative stress is indeed a complex process and while antioxidants may be beneficial in experimental settings where the disease model is well established, in the clinical setting where disease pathophysiology is confounded by different aetiologies and genetic heterogeneity, the use of antioxidants with undefined chemical composition could be inefficient or even harmful. Moreover, apart from general markers of oxidative stress (e.g. protein carbonylation, lipid peroxidation) more studies are needed to determine the specific proteins impacted by oxidative 
modifications (e.g. sarcomere proteins, ion channels) as this will form the impetus for identifying and developing more precise therapeutic interventions which could potentially lead to better clinical outcomes in patients with cardiac hypertrophy.

\section{FUNDING SOURCES}

Chrishan Ramachandra is supported by the Singapore Ministry of Health's National Medical Research Council under its Open Fund-Young Individual Research Grant (NMRC/OFYIRG/0073/2018), the National Health Innovation Centre Singapore under its Innovation to Develop Grant (NHIC-I2S-1811007) and the SingHealth Duke-NUS Academic Medical Centre under its SingHealth Duke-NUS Academic Medicine Research Grant (AM/TP033/2020 [SRDUKAMR2033]). Derek Hausenloy was supported by the British Heart Foundation (CS/14/3/31002), the Duke-NUS Signature Research Programme funded by the Ministry of Health, Singapore Ministry of Health's National Medical Research Council under its Clinician Scientist-Senior Investigator scheme (NMRC/CSA-SI/0011/2017), Centre Grant, and Collaborative Centre Grant scheme (NMRC/CGAug16C006). This article is based upon work from COST Action EU-CARDIOPROTECTION CA16225 supported by COST (European Cooperation in Science and Technology). 


\section{ABBREVIATIONS}

4E-BP1 Eukaryotic translation initiation factor 4E (elF4E)-binding protein 1

Akt Protein kinase $B$

AMPK AMP-activated protein kinase

ASK1 Apoptosis signal-regulating kinase 1

BCL10 B-cell lymphoma/leukemia 10

BIM Bcl-2-like protein 11

BP n-butylidenephthalide

BRD4 Bromodomain-containing protein 4

C1QTNF1 Complement C1q tumour necrosis factor-related protein 1

CaMKII Calmodulin-dependent protein kinase II

CAMP Cyclic adenosine monophosphate

CARD9 Caspase recruitment domain family member 9

CDC20 Cell division cycle protein 20 homolog

$\mathrm{CHCHD} 3$ Coiled-coil helix coiled-coil helix domain-containing protein 3

CHOP C/EBP homologous protein

CRAMP Cathelicidin-related antimicrobial peptide

CTRP3 C1q-tumour necrosis factor-related protein 3

CTRP9 C1q-tumour necrosis factor-related protein 9

Drp1 Dynamin-1-like protein

EGFR Epidermal growth factor receptor

EMP Endothelial microparticles

ENDOG Endonuclease $\mathrm{G}$

Erk1/2 Extracellular signal-regulated kinase 1/2

ERRa Estrogen-related receptor $\alpha$

FGF21 Fibroblast growth factor 21

Fis1 Mitochondrial fission 1 protein

FNDC5 Fibronectin type III domain containing 5

FOXO1 Forkhead box 01

FOXO3a Forkhead box O3

Gb3 Globotriaosylceramide

GLA Alpha-galactosidase

GLP-1 Glucagon-like peptide 1

GSK3 $3 \quad$ Glycogen synthase kinase 3 beta

$\mathrm{H}_{2} \mathrm{O}_{2} \quad$ Hydrogen peroxide

$\mathrm{H}_{2} \mathrm{~S} \quad H y d r o g e n$ sulfide

$\mathrm{H}_{2} \mathrm{Se} \quad H y d r o g e n$ selenide

hADSCs Human adipose-derived stem cells

HDAC4 Histone deacetylase 4

hESCs Human embryonic stem cells

HFD High-fat diet

HIF1a Hypoxia-inducible factor 1 alpha

HO-1 Heme oxygenase 1

Hsp22 Heat shock protein 22 


\begin{tabular}{|c|c|}
\hline IGFR1 & Insulin-like growth factor 1 \\
\hline IL-6 & Interleukin 6 \\
\hline isoLGs & Isolevuglandins \\
\hline JAK2 & Janus kinase 2 \\
\hline Jnk1/2 & C-Jun N-terminal kinases $1 / 2$ \\
\hline LC3 & Microtubule-associated proteins $1 \mathrm{~A} / 1 \mathrm{~B}$ light chain 3B \\
\hline LKB1 & Loss of tumor suppressor liver kinase B1 \\
\hline LOX & Lysyl oxidase \\
\hline LVH & Left ventricular hypertrophy \\
\hline MAPK & Mitogen-activated protein kinase \\
\hline mdivi-1 & Mitochondrial division inhibitor 1 \\
\hline MDM2 & Murine double minute 2 \\
\hline Mfn1 & Mitofusin 1 \\
\hline Mfn2 & Mitofusin 2 \\
\hline MICOS & Mitochondrial contact site and cristae organising system \\
\hline miRNA & Micro RNA \\
\hline mitoKATP & Mitochondrial ATP-sensitive potassium channel \\
\hline MitoQ & mitochondria-targeted ubiquinone \\
\hline MK2 & MAPK-activated protein kinase 2 \\
\hline MLP & Muscle LIM protein \\
\hline MnSOD & Manganese superoxide dismutase \\
\hline mtDNA & Mitochondrial DNA \\
\hline MTG1 & Mitochondrial ribosome associated GTPase 1 \\
\hline mTOR & Mammalian target of rapamycin \\
\hline NAC & N-acetyl cysteine \\
\hline $\mathrm{NaHS}$ & NaHS \\
\hline NCOA4 & Nuclear receptor coactivator 4 \\
\hline NF-kB & Nuclear factor kappa-light-chain-enhancer of activated B cells \\
\hline NHE1 & $\mathrm{Na}^{+} / \mathrm{H}^{+}$exchanger isoform 1 \\
\hline NKA & $\mathrm{Na}^{+} / \mathrm{K}^{+}-$ATPase \\
\hline NOX2 & NADPH oxidase 2 \\
\hline NOX4 & NADPH oxidase 4 \\
\hline NOX5 & NADPH oxidase 5 \\
\hline Nrf2 & Nuclear factor erythroid 2-related factor 2 \\
\hline Opa1 & Dynamin-like 120 kDa protein, mitochondrial \\
\hline p47phox & Neutrophil cytosol factor 1 \\
\hline PDE & Phosphodiesterase type 1 \\
\hline PDK4 & Pyruvate dehydrogenase kinase 4 \\
\hline PGC1a & Peroxisome proliferator activated receptor gamma coactivator 1 alpha \\
\hline PGC1 $\beta$ & Peroxisome proliferator activated receptor gamma coactivator 1 beta \\
\hline PI3K & Phosphoinositide 3-kinase \\
\hline PIKfyve & FYVE finger-containing phosphoinositide kinase \\
\hline PKA & Protein kinase A \\
\hline PLIN5 & Perilipin 5 \\
\hline
\end{tabular}




\begin{tabular}{|c|c|}
\hline PP2A & Protein phosphatase $2 \mathrm{~A}$ \\
\hline PP2Aca & Protein phosphatase $2 \mathrm{~A}$ catalytic subunit alpha \\
\hline PPARa & Peroxisome proliferator-activated receptor alpha \\
\hline PPARY & Peroxisome proliferator-activated receptor gamma \\
\hline PPARס & Peroxisome proliferator-activated receptor delta \\
\hline Rac1 & Ras-related C3 botulinum toxin substrate 1 \\
\hline RIP140 & Receptor-interacting protein of $140 \mathrm{kDa}$ \\
\hline ROCK-2 & Rho-associated coiled-coil containing protein kinase 2 \\
\hline RORa & Retinoid-related orphan nuclear receptor- $\alpha$ \\
\hline RPS6 & Ribosomal Protein S6 \\
\hline RS-LPS & Lipopolysaccharide from Rhodobacter sphaeroides \\
\hline SAM50 & Sorting and assembly machinery 50 \\
\hline SCAD & Short-chain acyl-CoA dehydrogenase \\
\hline SCLY & Selenocysteine lyase \\
\hline SFXN1 & Sideroflexin1 \\
\hline SIKE & Suppressor of IKK-epsilon \\
\hline SIRT1 & Sirtuin 1 \\
\hline SIRT3 & Sirtuin 3 \\
\hline SIRT4 & Sirtuin 4 \\
\hline STAT3 & Signal transducer and activator of transcription 3 \\
\hline TAK1 & Transforming growth factor beta-activated kinase 1 \\
\hline TBK1 & TANK-binding kinase 1 \\
\hline TGF $\beta 1$ & Transforming growth factor beta 1 \\
\hline TIM50 & Translocase of inner mitochondrial membrane 50 \\
\hline TLR4 & Toll-like receptor 4 \\
\hline TLR9 & Toll-like receptor 9 \\
\hline TRAF6 & Tumour necrosis factor receptor-associated factor 6 \\
\hline TRPC3 & Transient receptor potential channel, canonical 3 \\
\hline VDAC1 & Voltage-dependent anion-selective channel 1 \\
\hline$\alpha-C G R P$ & Calcitonin gene-related peptide \\
\hline
\end{tabular}




\section{REFERENCES}

[1] D. Mozaffarian, J.H. Caldwell, Right ventricular involvement in hypertrophic cardiomyopathy: a case report and literature review, Clin Cardiol 24(1) (2001) 2-8.

[2] E.D. Wigle, H. Rakowski, B.P. Kimball, W.G. Williams, Hypertrophic cardiomyopathy. Clinical spectrum and treatment, Circulation 92(7) (1995) 1680-92.

[3] S. Sasagawa, Y. Nishimura, S. Okabe, S. Murakami, Y. Ashikawa, M. Yuge, K. Kawaguchi, R. Kawase, R. Okamoto, M. Ito, T. Tanaka, Downregulation of GSTK1 Is a Common Mechanism Underlying Hypertrophic Cardiomyopathy, Front Pharmacol 7 (2016) 162.

[4] B. Quintana-Villamandos, L. Pazo-Sayos, I. Gonzalez Del Pozo, P. Rodriguez-Rodriguez, J.M. Bellon, A. Pedraz-Prieto, A.G. Pinto, M.C. Gonzalez, OXY-SCORE: a new perspective for left ventricular hypertrophy diagnosis, Ther Adv Chronic Dis 11 (2020) 2040622320936417.

[5] Z.M. Dimitrijevic, S.S. Salinger Martinovic, V.N. Nikolic, T.P. Cvetkovic, Protein Carbonyl Content Is a Predictive Biomarker of Eccentric Left Ventricular Hypertrophy in Hemodialysis Patients, Diagnostics (Basel) 9(4) (2019).

[6] D. Drozdz, P. Kwinta, K. Sztefko, Z. Kordon, T. Drozdz, M. Latka, M. Miklaszewska, K. Zachwieja, A. Rudzinski, J.A. Pietrzyk, Oxidative Stress Biomarkers and Left Ventricular Hypertrophy in Children with Chronic Kidney Disease, Oxid Med Cell Longev 2016 (2016) 7520231.

[7] W. Dröge, Free radicals in the physiological control of cell function, Physiol Rev 82(1) (2002) 47-95.

[8] T. Finkel, Signal transduction by reactive oxygen species, J Cell Biol 194(1) (2011) 7-15.

[9] K. Raedschelders, D.M. Ansley, D.D. Chen, The cellular and molecular origin of reactive oxygen species generation during myocardial ischemia and reperfusion, Pharmacol Ther 133(2) (2012) 23055.

[10] H. Sies, C. Berndt, D.P. Jones, Oxidative Stress, Annu Rev Biochem 86 (2017) 715-748.

[11] M. Seddon, Y.H. Looi, A.M. Shah, Oxidative stress and redox signalling in cardiac hypertrophy and heart failure, Heart 93(8) (2007) 903-7.

[12] A. van der Pol, W.H. van Gilst, A.A. Voors, P. van der Meer, Treating oxidative stress in heart failure: past, present and future, Eur J Heart Fail 21(4) (2019) 425-435.

[13] A.D. Fotino, A.M. Thompson-Paul, L.A. Bazzano, Effect of coenzyme $Q_{10}$ supplementation on heart failure: a meta-analysis, Am J Clin Nutr 97(2) (2013) 268-75.

[14] A. Konior, A. Schramm, M. Czesnikiewicz-Guzik, T.J. Guzik, NADPH oxidases in vascular pathology, Antioxid Redox Signal 20(17) (2014) 2794-814.

[15] A.P. Harvey, E. Robinson, K.S. Edgar, R. McMullan, K.M. O'Neill, M. Alderdice, R. Amirkhah, P.D. Dunne, B.J. McDermott, D.J. Grieve, Downregulation of PPARalpha during Experimental Left Ventricular Hypertrophy Is Critically Dependent on Nox2 NADPH Oxidase Signalling, Int J Mol Sci 21(12) (2020).

[16] D. Kar, A. Bandyopadhyay, Targeting Peroxisome Proliferator Activated Receptor alpha (PPAR alpha) for the Prevention of Mitochondrial Impairment and Hypertrophy in Cardiomyocytes, Cell Physiol Biochem 49(1) (2018) 245-259.

[17] T. Ago, J. Kuroda, J. Pain, C. Fu, H. Li, J. Sadoshima, Upregulation of Nox4 by hypertrophic stimuli promotes apoptosis and mitochondrial dysfunction in cardiac myocytes, Circ Res 106(7) (2010) 125364.

[18] J. Kuroda, T. Ago, S. Matsushima, P. Zhai, M.D. Schneider, J. Sadoshima, NADPH oxidase 4 (Nox4) is a major source of oxidative stress in the failing heart, Proc Natl Acad Sci U S A 107(35) (2010) 1556570.

[19] K. Okabe, S. Matsushima, S. Ikeda, M. Ikeda, A. Ishikita, T. Tadokoro, N. Enzan, T. Yamamoto, M. Sada, H. Deguchi, K. Shinohara, T. Ide, H. Tsutsui, DPP (Dipeptidyl Peptidase)-4 Inhibitor Attenuates Ang II (Angiotensin II)-Induced Cardiac Hypertrophy via GLP (Glucagon-Like Peptide)-1-Dependent Suppression of Nox (Nicotinamide Adenine Dinucleotide Phosphate Oxidase) 4-HDAC (Histone Deacetylase) 4 Pathway, Hypertension 75(4) (2020) 991-1001.

[20] M. Zhang, A.C. Brewer, K. Schroder, C.X. Santos, D.J. Grieve, M. Wang, N. Anilkumar, B. Yu, X. Dong, S.J. Walker, R.P. Brandes, A.M. Shah, NADPH oxidase-4 mediates protection against chronic 
load-induced stress in mouse hearts by enhancing angiogenesis, Proc Natl Acad Sci U S A 107(42) (2010) 18121-6.

[21] M. Schnelle, I. Sawyer, N. Anilkumar, B.A. Mohamed, D.A. Richards, K. Toischer, M. Zhang, N. Catibog, G. Sawyer, H. Mongue-Din, K. Schroder, G. Hasenfuss, A.M. Shah, NADPH oxidase-4 promotes eccentric cardiac hypertrophy in response to volume overload, Cardiovasc Res (2019).

[22] A. Nabeebaccus, A. Hafstad, T. Eykyn, X. Yin, A. Brewer, M. Zhang, M. Mayr, A. Shah, Cardiactargeted NADPH oxidase 4 in the adaptive cardiac remodelling of the murine heart, Lancet 385 Suppl 1 (2015) S73.

[23] M. Manti, R. Fornes, G. Pironti, S. McCann Haworth, Z. Zhengbing, A. Benrick, M. Carlstrom, D. Andersson, E. Stener-Victorin, Maternal androgen excess induces cardiac hypertrophy and left ventricular dysfunction in female mice offspring, Cardiovasc Res 116(3) (2020) 619-632.

[24] G.J. Zhao, C.L. Zhao, S. Ouyang, K.Q. Deng, L. Zhu, A.C. Montezano, C. Zhang, F. Hu, X.Y. Zhu, S. Tian, X. Liu, Y.X. Ji, P. Zhang, X.J. Zhang, Z.G. She, R.M. Touyz, H. Li, Ca(2+)-Dependent NOX5 (NADPH Oxidase 5) Exaggerates Cardiac Hypertrophy Through Reactive Oxygen Species Production, Hypertension 76(3) (2020) 827-838.

[25] M. Galan, S. Varona, A. Guadall, M. Orriols, M. Navas, S. Aguilo, A. de Diego, M.A. Navarro, D. Garcia-Dorado, A. Rodriguez-Sinovas, J. Martinez-Gonzalez, C. Rodriguez, Lysyl oxidase overexpression accelerates cardiac remodeling and aggravates angiotensin II-induced hypertrophy, FASEB J 31(9) (2017) 3787-3799.

[26] J.A. Byrne, D.J. Grieve, J.K. Bendall, J.M. Li, C. Gove, J.D. Lambeth, A.C. Cave, A.M. Shah, Contrasting roles of NADPH oxidase isoforms in pressure-overload versus angiotensin II-induced cardiac hypertrophy, Circ Res 93(9) (2003) 802-5.

[27] U. Novoa, D. Arauna, M. Moran, M. Nunez, S. Zagmutt, S. Saldivia, C. Valdes, J. Villasenor, C.G. Zambrano, D.R. Gonzalez, High-Intensity Exercise Reduces Cardiac Fibrosis and Hypertrophy but Does Not Restore the Nitroso-Redox Imbalance in Diabetic Cardiomyopathy, Oxid Med Cell Longev 2017 (2017) 7921363.

[28] S.N. Bhatti, J.M. Li, Nox2 dependent redox-regulation of Akt and ERK1/2 to promote left ventricular hypertrophy in dietary obesity of mice, Biochem Biophys Res Commun 528(3) (2020) 506513.

[29] S. Wang, J. Gu, Z. Xu, Z. Zhang, T. Bai, J. Xu, J. Cai, G. Barnes, Q.J. Liu, J.H. Freedman, Y. Wang, Q. Liu, Y. Zheng, L. Cai, Zinc rescues obesity-induced cardiac hypertrophy via stimulating metallothionein to suppress oxidative stress-activated BCL10/CARD9/p38 MAPK pathway, J Cell Mol Med 21(6) (2017) $1182-1192$.

[30] A. Zuo, X. Zhao, T. Li, J. Li, S. Lei, J. Chen, D. Xu, C. Song, T. Liu, C. Li, Y. Guo, CTRP9 knockout exaggerates lipotoxicity in cardiac myocytes and high-fat diet-induced cardiac hypertrophy through inhibiting the LKB1/AMPK pathway, J Cell Mol Med 24(4) (2020) 2635-2647.

[31] R.Q. Xue, M. Zhao, Q. Wu, S. Yang, Y.L. Cui, X.J. Yu, J. Liu, W.J. Zang, Regulation of mitochondrial cristae remodelling by acetylcholine alleviates palmitate-induced cardiomyocyte hypertrophy, Free Radic Biol Med 145 (2019) 103-117.

[32] H. Tronchere, M. Cinato, A. Timotin, L. Guitou, C. Villedieu, H. Thibault, D. Baetz, B. Payrastre, P. Valet, A. Parini, O. Kunduzova, F. Boal, Inhibition of PIKfyve prevents myocardial apoptosis and hypertrophy through activation of SIRT3 in obese mice, EMBO Mol Med 9(6) (2017) 770-785.

[33] J. You, Z. Yue, S. Chen, Y. Chen, X. Lu, X. Zhang, P. Shen, J. Li, Q. Han, Z. Li, P. Liu, Receptorinteracting Protein 140 represses Sirtuin 3 to facilitate hypertrophy, mitochondrial dysfunction and energy metabolic dysfunction in cardiomyocytes, Acta Physiol (Oxf) 220(1) (2017) 58-71.

[34] C. Wang, Y. Yuan, J. Wu, Y. Zhao, X. Gao, Y. Chen, C. Sun, L. Xiao, P. Zheng, P. Hu, Z. Li, Z. Wang, J. Ye, L. Zhang, Plin5 deficiency exacerbates pressure overload-induced cardiac hypertrophy and heart failure by enhancing myocardial fatty acid oxidation and oxidative stress, Free Radic Biol Med 141 (2019) 372-382. 
[35] K.C. Cheng, W.T. Chang, Y. Li, Y.Z. Cheng, J.T. Cheng, Z.C. Chen, GW0742 activates peroxisome proliferator-activated receptor delta to reduce free radicals and alleviate cardiac hypertrophy induced by hyperglycemia in cultured H9c2 cells, J Cell Biochem 119(11) (2018) 9532-9542.

[36] H. Li, C. Xu, Q. Li, X. Gao, E. Sugano, H. Tomita, L. Yang, S. Shi, Thioredoxin 2 Offers Protection against Mitochondrial Oxidative Stress in H9c2 Cells and against Myocardial Hypertrophy Induced by Hyperglycemia, Int J Mol Sci 18(9) (2017).

[37] J. Li, B.A. Kemp, N.L. Howell, J. Massey, K. Minczuk, Q. Huang, M.D. Chordia, R.J. Roy, J.T. Patrie, G.E. Davogustto, C.M. Kramer, F.H. Epstein, R.M. Carey, H. Taegtmeyer, S.R. Keller, B.K. Kundu, Metabolic Changes in Spontaneously Hypertensive Rat Hearts Precede Cardiac Dysfunction and Left Ventricular Hypertrophy, J Am Heart Assoc 8(4) (2019) e010926.

[38] J. Li, K. Minczuk, J.C. Massey, N.L. Howell, R.J. Roy, S. Paul, J.T. Patrie, C.M. Kramer, F.H. Epstein, R.M. Carey, H. Taegtmeyer, S.R. Keller, B.K. Kundu, Metformin Improves Cardiac Metabolism and Function, and Prevents Left Ventricular Hypertrophy in Spontaneously Hypertensive Rats, J Am Heart Assoc 9(7) (2020) e015154.

[39] M. Mohan, S. Al-Talabany, A. McKinnie, I.R. Mordi, J.S.S. Singh, S.J. Gandy, F. Baig, M.S. Hussain, U. Bhalraam, F. Khan, A.M. Choy, S. Matthew, J.G. Houston, A.D. Struthers, J. George, C.C. Lang, A randomized controlled trial of metformin on left ventricular hypertrophy in patients with coronary artery disease without diabetes: the MET-REMODEL trial, Eur Heart J 40(41) (2019) 3409-3417.

[40] J. Huang, L. Xu, Q. Huang, J. Luo, P. Liu, S. Chen, X. Yuan, Y. Lu, P. Wang, S. Zhou, Changes in shortchain acyl-coA dehydrogenase during rat cardiac development and stress, J Cell Mol Med 19(7) (2015) 1672-88.

[41] Z. Ma, X. Qin, X. Zhong, Y. Liao, Y. Su, X. Liu, P. Liu, J. Lu, S. Zhou, Flavine adenine dinucleotide inhibits pathological cardiac hypertrophy and fibrosis through activating short chain acyl-CoA dehydrogenase, Biochem Pharmacol 178 (2020) 114100.

[42] S.A. Mohammed, B. Paramesha, Y. Kumar, U. Tariq, S.K. Arava, S.K. Banerjee, Allylmethylsulfide, a Sulfur Compound Derived from Garlic, Attenuates Isoproterenol-Induced Cardiac Hypertrophy in Rats, Oxid Med Cell Longev 2020 (2020) 7856318.

[43] T.N. Khatua, R.M. Borkar, S.A. Mohammed, A.K. Dinda, R. Srinivas, S.K. Banerjee, Novel Sulfur Metabolites of Garlic Attenuate Cardiac Hypertrophy and Remodeling through Induction of $\mathrm{Na}(+) / K(+)$-ATPase Expression, Front Pharmacol 8 (2017) 18.

[44] H.S. An, J.Y. Lee, E.B. Choi, E.A. Jeong, H.J. Shin, K.E. Kim, K.A. Park, Z. Jin, J.E. Lee, J.S. Koh, W. Kwak, W.H. Kim, G.S. Roh, Caloric restriction reverses left ventricular hypertrophy through the regulation of cardiac iron homeostasis in impaired leptin signaling mice, Sci Rep 10(1) (2020) 7176.

[45] C.E.B. David, A.M.B. Lucas, M.T.S. Araujo, B.N. Coelho, J.B.S. Neto, B.R.C. Portela, A.L.N. Varela, A.J. Kowaltowski, H.T. Facundo, Calorie restriction attenuates hypertrophy-induced redox imbalance and mitochondrial ATP-sensitive K(+) channel repression, J Nutr Biochem 62 (2018) 87-94.

[46] M. Lombardi, D. Lazzeroni, A. Pisano, F. Girolami, O. Alfieri, G. La Canna, G. d'Amati, I. Olivotto, O.E. Rimoldi, C. Foglieni, P.G. Camici, Mitochondrial Energetics and Ca2(+)-Activated ATPase in Obstructive Hypertrophic Cardiomyopathy, J Clin Med 9(6) (2020).

[47] A. Mitra, R. Datta, S. Rana, S. Sarkar, Modulation of NFKB1/p50 by ROS leads to impaired ATP production during MI compared to cardiac hypertrophy, J Cell Biochem 119(2) (2018) 1575-1590.

[48] M. Tang, Z. Huang, X. Luo, M. Liu, L. Wang, Z. Qi, S. Huang, J. Zhong, J.X. Chen, L. Li, D. Wu, L. Chen, Ferritinophagy activation and sideroflexin1-dependent mitochondria iron overload is involved in apelin-13-induced cardiomyocytes hypertrophy, Free Radic Biol Med 134 (2019) 445-457.

[49] D.F. Dai, T. Chen, J. Wanagat, M. Laflamme, D.J. Marcinek, M.J. Emond, C.P. Ngo, T.A. Prolla, P.S. Rabinovitch, Age-dependent cardiomyopathy in mitochondrial mutator mice is attenuated by overexpression of catalase targeted to mitochondria, Aging Cell 9(4) (2010) 536-44.

[50] N. Blasco, Y. Camara, E. Nunez, A. Bea, G. Bares, C. Forne, M. Ruiz-Meana, C. Giron, I. Barba, E. Garcia-Arumi, D. Garcia-Dorado, J. Vazquez, R. Marti, M. Llovera, D. Sanchis, Cardiomyocyte hypertrophy induced by Endonuclease $\mathrm{G}$ deficiency requires reactive oxygen radicals accumulation and is inhibitable by the micropeptide humanin, Redox Biol 16 (2018) 146-156. 
[51] D. Xu, Y. Zhao, X. Weng, Y. Lu, W. Li, K. Tang, W. Chen, Z. Liu, X. Qi, J. Zheng, J. Fassett, Y. Zhang, $\mathrm{Y}$. Xu, Novel role of mitochondrial GTPases 1 in pathological cardiac hypertrophy, J Mol Cell Cardiol 128 (2019) 105-116.

[52] K. Tang, Y. Zhao, H. Li, M. Zhu, W. Li, W. Liu, G. Zhu, D. Xu, W. Peng, Y.W. Xu, Translocase of Inner Membrane 50 Functions as a Novel Protective Regulator of Pathological Cardiac Hypertrophy, J Am Heart Assoc 6(4) (2017).

[53] Y.L. Fu, L. Tao, F.H. Peng, N.Z. Zheng, Q. Lin, S.Y. Cai, Q. Wang, GJA1-20k attenuates Ang II-induced pathological cardiac hypertrophy by regulating gap junction formation and mitochondrial function, Acta Pharmacol Sin (2020).

[54] D. Graham, N.N. Huynh, C.A. Hamilton, E. Beattie, R.A. Smith, H.M. Cocheme, M.P. Murphy, A.F. Dominiczak, Mitochondria-targeted antioxidant MitoQ10 improves endothelial function and attenuates cardiac hypertrophy, Hypertension 54(2) (2009) 322-8.

[55] J. McLachlan, E. Beattie, M.P. Murphy, C.H. Koh-Tan, E. Olson, W. Beattie, A.F. Dominiczak, S.A. Nicklin, D. Graham, Combined therapeutic benefit of mitochondria-targeted antioxidant, MitoQ10, and angiotensin receptor blocker, losartan, on cardiovascular function, J Hypertens 32(3) (2014) 55564.

[56] P. Hasan, M. Saotome, T. Ikoma, K. Iguchi, H. Kawasaki, T. Iwashita, H. Hayashi, Y. Maekawa, Mitochondrial fission protein, dynamin-related protein 1, contributes to the promotion of hypertensive cardiac hypertrophy and fibrosis in Dahl-salt sensitive rats, J Mol Cell Cardiol 121 (2018) 103-106.

[57] L. Fang, A.H. Ellims, A.L. Beale, A.J. Taylor, A. Murphy, A.M. Dart, Systemic inflammation is associated with myocardial fibrosis, diastolic dysfunction, and cardiac hypertrophy in patients with hypertrophic cardiomyopathy, Am J Transl Res 9(11) (2017) 5063-5073.

[58] Y. Yang, J. Lv, S. Jiang, Z. Ma, D. Wang, W. Hu, C. Deng, C. Fan, S. Di, Y. Sun, W. Yi, The emerging role of Toll-like receptor 4 in myocardial inflammation, Cell Death Dis 7(5) (2016) e2234.

[59] M.O.R. Mian, Y. He, M. Bertagnolli, T.A. Mai-Vo, R.O. Fernandes, F. Boudreau, A. Cloutier, T.M. Luu, A.M. Nuyt, TLR (Toll-Like Receptor) 4 Antagonism Prevents Left Ventricular Hypertrophy and Dysfunction Caused by Neonatal Hyperoxia Exposure in Rats, Hypertension 74(4) (2019) 843-853.

[60] P.B. Katare, P.K. Bagul, A.K. Dinda, S.K. Banerjee, Toll-Like Receptor 4 Inhibition Improves Oxidative Stress and Mitochondrial Health in Isoproterenol-Induced Cardiac Hypertrophy in Rats, Front Immunol 8 (2017) 719.

[61] D. Chen, Z. Li, P. Bao, M. Chen, M. Zhang, F. Yan, Y. Xu, C. Ji, X. Hu, D. Sanchis, Y. Zhang, J. Ye, Nrf2 deficiency aggravates Angiotensin II-induced cardiac injury by increasing hypertrophy and enhancing IL-6/STAT3-dependent inflammation, Biochim Biophys Acta Mol Basis Dis 1865(6) (2019) 1253-1264.

[62] C. Tang, G. Yin, C. Huang, H. Wang, J. Gao, J. Luo, Z. Zhang, J. Wang, J. Hong, X. Chai, Peroxiredoxin1 ameliorates pressure overload-induced cardiac hypertrophy and fibrosis, Biomed Pharmacother 129 (2020) 110357.

[63] W. Zhu, R.D. Wu, Y.G. Lv, Y.M. Liu, H. Huang, J.Q. Xu, BRD4 blockage alleviates pathological cardiac hypertrophy through the suppression of fibrosis and inflammation via reducing ROS generation, Biomed Pharmacother 121 (2020) 109368.

[64] Z.G. Ma, Y.P. Yuan, X. Zhang, S.C. Xu, C.Y. Kong, P. Song, N. Li, Q.Z. Tang, C1q-tumour necrosis factor-related protein-3 exacerbates cardiac hypertrophy in mice, Cardiovasc Res $115(6)$ (2019) 1067 1077.

[65] Z. Geng, W.Y. Fan, B. Zhou, C. Ye, Y. Tong, Y.B. Zhou, X.Q. Xiong, FNDC5 attenuates obesityinduced cardiac hypertrophy by inactivating JAK2/STAT3-associated inflammation and oxidative stress, J Transl Med 17(1) (2019) 107.

[66] S. Li, Z. Zhu, M. Xue, X. Yi, J. Liang, C. Niu, G. Chen, Y. Shen, H. Zhang, J. Zheng, C. Zhao, Y. Liang, W. Cong, Y. Wang, L. Jin, Fibroblast growth factor 21 protects the heart from angiotensin II-induced cardiac hypertrophy and dysfunction via SIRT1, Biochim Biophys Acta Mol Basis Dis 1865(6) (2019) 1241-1252. 
[67] A. Schäffler, C. Buechler, CTRP family: linking immunity to metabolism, Trends Endocrinol Metab 23(4) (2012) 194-204.

[68] L. Wu, L. Gao, D. Zhang, R. Yao, Z. Huang, B. Du, Z. Wang, L. Xiao, P. Li, Y. Li, C. Liang, Y. Zhang, C1QTNF1 attenuates angiotensin II-induced cardiac hypertrophy via activation of the AMPKa pathway, Free Radic Biol Med 121 (2018) 215-230.

[69] D. Tripathi, B. Biswas, A. Manhas, A. Singh, D. Goyal, M. Gaestel, K. Jagavelu, Proinflammatory Effect of Endothelial Microparticles Is Mitochondria Mediated and Modulated Through MAPKAPK2 (MAPK-Activated Protein Kinase 2) Leading to Attenuation of Cardiac Hypertrophy, Arterioscler Thromb Vasc Biol 39(6) (2019) 1100-1112.

[70] B. Butts, D.A. Calhoun, T.S. Denney, Jr., S.G. Lloyd, H. Gupta, K.K. Gaddam, I. Aban, S. Oparil, P.W. Sanders, R. Patel, J.F. Collawn, L.J. Dell'Italia, Plasma xanthine oxidase activity is related to increased sodium and left ventricular hypertrophy in resistant hypertension, Free Radic Biol Med 134 (2019) 343-349.

[71] Y.P. Xie, S. Lai, Q.Y. Lin, X. Xie, J.W. Liao, H.X. Wang, C. Tian, H.H. Li, CDC20 regulates cardiac hypertrophy via targeting LC3-dependent autophagy, Theranostics 8(21) (2018) 5995-6007.

[72] C. Kampmann, A. Linhart, F. Baehner, T. Palecek, C.M. Wiethoff, E. Miebach, C. Whybra, A. Gal, J. Bultas, M. Beck, Onset and progression of the Anderson-Fabry disease related cardiomyopathy, Int J Cardiol 130(3) (2008) 367-73.

[73] H.Y. Song, C.S. Chien, A.A. Yarmishyn, S.J. Chou, Y.P. Yang, M.L. Wang, C.Y. Wang, H.B. Leu, W.C. Yu, Y.L. Chang, S.H. Chiou, Generation of GLA-Knockout Human Embryonic Stem Cell Lines to Model Autophagic Dysfunction and Exosome Secretion in Fabry Disease-Associated Hypertrophic Cardiomyopathy, Cells 8(4) (2019).

[74] M. Xu, C.X. Wan, S.H. Huang, H.B. Wang, D. Fan, H.M. Wu, Q.Q. Wu, Z.G. Ma, W. Deng, Q.Z. Tang, Oridonin protects against cardiac hypertrophy by promoting P21-related autophagy, Cell Death Dis 10(6) (2019) 403.

[75] T.T. Cao, H.H. Chen, Z. Dong, Y.W. Xu, P. Zhao, W. Guo, H.C. Wei, C. Zhang, R. Lu, Stachydrine Protects Against Pressure Overload-Induced Cardiac Hypertrophy by Suppressing Autophagy, Cell Physiol Biochem 42(1) (2017) 103-114.

[76] F. Xie, D. Wu, S.F. Huang, J.G. Cao, H.N. Li, L. He, M.Q. Liu, L.F. Li, L.X. Chen, The endoplasmic reticulum stress-autophagy pathway is involved in apelin-13-induced cardiomyocyte hypertrophy in vitro, Acta Pharmacol Sin 38(12) (2017) 1589-1600.

[77] Y.X. Ji, P. Zhang, X.J. Zhang, Y.C. Zhao, K.Q. Deng, X. Jiang, P.X. Wang, Z. Huang, H. Li, The ubiquitin E3 ligase TRAF6 exacerbates pathological cardiac hypertrophy via TAK1-dependent signalling, Nat Commun 7 (2016) 11267.

[78] P. Zhou, REGgamma: a shortcut to destruction, Cell 124(2) (2006) 256-7.

[79] Y. Xie, Y. Gao, R. Gao, W. Yang, Z. Dong, R.E. Moses, A. Sun, X. Li, J. Ge, The proteasome activator REGgamma accelerates cardiac hypertrophy by declining PP2Acalpha-SOD2 pathway, Cell Death Differ 27(10) (2020) 2952-2972.

[80] D. Morin, R. Long, M. Panel, L. Laure, A. Taranu, C. Gueguen, S. Pons, V. Leoni, C. Caccia, S.F. Vatner, D.E. Vatner, H. Qiu, C. Depre, A. Berdeaux, B. Ghaleh, Hsp22 overexpression induces myocardial hypertrophy, senescence and reduced life span through enhanced oxidative stress, Free Radic Biol Med 137 (2019) 194-200.

[81] J. Acunzo, M. Katsogiannou, P. Rocchi, Small heat shock proteins HSP27 (HspB1), aB-crystallin (HspB5) and HSP22 (HspB8) as regulators of cell death, Int J Biochem Cell Biol 44(10) (2012) 1622-31. [82] Y. Liu, J. Afzal, S. Vakrou, G.V. Greenland, C.C. Talbot, Jr., V.B. Hebl, Y. Guan, R. Karmali, J.C. Tardiff, L.A. Leinwand, J.E. Olgin, S. Das, R. Fukunaga, M.R. Abraham, Differences in microRNA-29 and Profibrotic Gene Expression in Mouse and Human Hypertrophic Cardiomyopathy, Front Cardiovasc Med 6 (2019) 170.

[83] S. Hu, M. Cheng, X. Guo, S. Wang, B. Liu, H. Jiang, C. Huang, G. Wu, Down-regulation of miR-200c attenuates Angll-induced cardiac hypertrophy via targeting the MLCK-mediated pathway, J Cell Mol Med 23(4) (2019) 2505-2516. 
[84] X. Guan, L. Wang, Z. Liu, X. Guo, Y. Jiang, Y. Lu, Y. Peng, T. Liu, B. Yang, H. Shan, Y. Zhang, C. Xu, miR-106a promotes cardiac hypertrophy by targeting mitofusin 2, J Mol Cell Cardiol 99 (2016) 207217.

[85] J.A. Hill, Electrical remodeling in cardiac hypertrophy, Trends Cardiovasc Med 13(8) (2003) 31622.

[86] M.S. Brea, R.G. Diaz, D.S. Escudero, M.R. Zavala, E.L. Portiansky, M.C. Villa-Abrille, C.I. Caldiz, N.G. Perez, P.E. Morgan, Silencing of epidermal growth factor receptor reduces $\mathrm{Na}(+) / \mathrm{H}(+)$ exchanger 1 activity and hypertensive cardiac hypertrophy, Biochem Pharmacol 170 (2019) 113667.

[87] S. Xiong, H.J. Sun, L. Cao, M. Zhu, T. Liu, Z. Wu, J.S. Bian, Stimulation of $\mathrm{Na}(+) / \mathrm{K}(+)-A T P a s e$ with an Antibody against Its 4(th) Extracellular Region Attenuates Angiotensin II-Induced H9c2 Cardiomyocyte Hypertrophy via an AMPK/SIRT3/PPARgamma Signaling Pathway, Oxid Med Cell Longev 2019 (2019) 4616034.

[88] T.Y. Kim, R. Terentyeva, K.H. Roder, W. Li, M. Liu, I. Greener, S. Hamilton, I. Polina, K.R. Murphy, R.T. Clements, S.C. Dudley, Jr., G. Koren, B.R. Choi, D. Terentyev, SK channel enhancers attenuate $\mathrm{Ca2+-dependent} \mathrm{arrhythmia} \mathrm{in} \mathrm{hypertrophic} \mathrm{hearts} \mathrm{by} \mathrm{regulating} \mathrm{mito-ROS-dependent} \mathrm{oxidation} \mathrm{and}$ activity of RyR, Cardiovasc Res 113(3) (2017) 343-353.

[89] X. Li, W.J. Lu, Y. Li, F. Wu, R. Bai, S. Ma, T. Dong, H. Zhang, A.S. Lee, Y. Wang, F. Lan, MLP-deficient human pluripotent stem cell derived cardiomyocytes develop hypertrophic cardiomyopathy and heart failure phenotypes due to abnormal calcium handling, Cell Death Dis 10(8) (2019) 610.

[90] S. Kumari, P.B. Katare, R. Elancheran, H.L. Nizami, B. Paramesha, S. Arava, P.P. Sarma, R. Kumar, D. Mahajan, Y. Kumar, R. Devi, S.K. Banerjee, Musa balbisiana Fruit Rich in Polyphenols Attenuates Isoproterenol-Induced Cardiac Hypertrophy in Rats via Inhibition of Inflammation and Oxidative Stress, Oxid Med Cell Longev 2020 (2020) 7147498.

[91] R.A. Eladwy, E.M. Mantawy, W.M. El-Bakly, M. Fares, L.A. Ramadan, S.S. Azab, Mechanistic insights to the cardioprotective effect of blueberry nutraceutical extract in isoprenaline-induced cardiac hypertrophy, Phytomedicine 51 (2018) 84-93.

[92] G. Kumar, N. Saleem, S. Kumar, S.K. Maulik, S. Ahmad, M. Sharma, S.K. Goswami, Transcriptomic Validation of the Protective Effects of Aqueous Bark Extract of Terminalia arjuna (Roxb.) on Isoproterenol-Induced Cardiac Hypertrophy in Rats, Front Pharmacol 10 (2019) 1443.

[93] M. Al Hariri, K. Zibara, W. Farhat, Y. Hashem, N. Soudani, F. Al Ibrahim, E. Hamade, A. Zeidan, A. Husari, F. Kobeissy, Cigarette Smoking-Induced Cardiac Hypertrophy, Vascular Inflammation and Injury Are Attenuated by Antioxidant Supplementation in an Animal Model, Front Pharmacol 7 (2016) 397.

[94] M. Zhai, Z. Liu, B. Zhang, L. Jing, B. Li, K. Li, X. Chen, M. Zhang, B. Yu, K. Ren, Y. Yang, W. Yi, J. Yang, J. Liu, D. Yi, H. Liang, Z. Jin, R.J. Reiter, W. Duan, S. Yu, Melatonin protects against the pathological cardiac hypertrophy induced by transverse aortic constriction through activating PGC-1beta: In vivo and in vitro studies, J Pineal Res 63(3) (2017).

[95] N. Zhang, W.Y. Wei, Z. Yang, Y. Che, Y.G. Jin, H.H. Liao, S.S. Wang, W. Deng, Q.Z. Tang, Nobiletin, a Polymethoxy Flavonoid, Protects Against Cardiac Hypertrophy Induced by Pressure-Overload via Inhibition of NAPDH Oxidases and Endoplasmic Reticulum Stress, Cell Physiol Biochem 42(4) (2017) 1313-1325.

[96] Y. Chen, H.Q. Luo, L.L. Sun, M.T. Xu, J. Yu, L.L. Liu, J.Y. Zhang, Y.Q. Wang, H.X. Wang, X.F. Bao, G.L. Meng, Dihydromyricetin Attenuates Myocardial Hypertrophy Induced by Transverse Aortic Constriction via Oxidative Stress Inhibition and SIRT3 Pathway Enhancement, Int J Mol Sci 19(9) (2018). [97] Y.X. Luo, X. Tang, X.Z. An, X.M. Xie, X.F. Chen, X. Zhao, D.L. Hao, H.Z. Chen, D.P. Liu, SIRT4 accelerates Ang II-induced pathological cardiac hypertrophy by inhibiting manganese superoxide dismutase activity, Eur Heart J 38(18) (2017) 1389-1398.

[98] Y. Chen, Z. Ge, S. Huang, L. Zhou, C. Zhai, Y. Chen, Q. Hu, W. Cao, Y. Weng, Y. Li, Delphinidin attenuates pathological cardiac hypertrophy via the AMPK/NOX/MAPK signaling pathway, Aging (Albany NY) 12(6) (2020) 5362-5383. 
[99] B. Dong, C. Liu, R. Xue, Y. Wang, Y. Sun, Z. Liang, W. Fan, J. Jiang, J. Zhao, Q. Su, G. Dai, Y. Dong, H. Huang, Fisetin inhibits cardiac hypertrophy by suppressing oxidative stress, J Nutr Biochem 62 (2018) 221-229.

[100] P. Nie, F. Meng, J. Zhang, X. Wei, C. Shen, Astragaloside IV Exerts a Myocardial Protective Effect against Cardiac Hypertrophy in Rats, Partially via Activating the Nrf2/HO-1 Signaling Pathway, Oxid Med Cell Longev 2019 (2019) 4625912.

[101] Z.H. Liu, H.B. Liu, J. Wang, Astragaloside IV protects against the pathological cardiac hypertrophy in mice, Biomed Pharmacother 97 (2018) 1468-1478.

[102] S.Y. Zeng, L. Yang, H.Q. Lu, Q.J. Yan, L. Gao, X.P. Qin, Rutaecarpine prevents hypertensive cardiac hypertrophy involving the inhibition of Nox4-ROS-ADAM17 pathway, J Cell Mol Med 23(6) (2019) 4196-4207.

[103] N. Zhang, X. An, P. Lang, F. Wang, Y. Xie, Ginsenoside Rd contributes the attenuation of cardiac hypertrophy in vivo and in vitro, Biomed Pharmacother 109 (2019) 1016-1023.

[104] D. Dos Santos Lacerda, P. Turck, B. Gazzi de Lima-Seolin, R. Colombo, V. Duarte Ortiz, J.H. Poletto Bonetto, C. Campos-Carraro, S.E. Bianchi, A. Bello-Klein, V. Linck Bassani, A. Sander da Rosa Araujo, Pterostilbene reduces oxidative stress, prevents hypertrophy and preserves systolic function of right ventricle in cor pulmonale model, Br J Pharmacol 174(19) (2017) 3302-3314.

[105] J. Zeng, J. Zhao, B. Dong, X. Cai, J. Jiang, R. Xue, F. Yao, Y. Dong, C. Liu, Lycopene protects against pressure overload-induced cardiac hypertrophy by attenuating oxidative stress, J Nutr Biochem 66 (2019) 70-78.

[106] F. Liu, H. Su, B. Liu, Y. Mei, Q. Ke, X. Sun, W. Tan, STVNa Attenuates Isoproterenol-Induced Cardiac Hypertrophy Response through the HDAC4 and Prdx2/ROS/Trx1 Pathways, Int J Mol Sci 21(2) (2020).

[107] L.K. Cole, E.M. Mejia, G.C. Sparagna, M. Vandel, B. Xiang, X. Han, N. Dedousis, B.A. Kaufman, V.W. Dolinsky, G.M. Hatch, Cardiolipin deficiency elevates susceptibility to a lipotoxic hypertrophic cardiomyopathy, J Mol Cell Cardiol 144 (2020) 24-34.

[108] A.J. Marian, Y. Tan, L. Li, J. Chang, P. Syrris, M. Hessabi, M.H. Rahbar, J.T. Willerson, B.Y. Cheong, C.Y. Liu, N.S. Kleiman, D.A. Bluemke, S.F. Nagueh, Hypertrophy Regression With N-Acetylcysteine in Hypertrophic Cardiomyopathy (HALT-HCM): A Randomized, Placebo-Controlled, Double-Blind Pilot Study, Circ Res 122(8) (2018) 1109-1118.

[109] D.R.A. Reyes, M.J. Gomes, C.M. Rosa, L.U. Pagan, F.C. Damatto, R.L. Damatto, I. Depra, D.H.S. Campos, A.A.H. Fernandez, P.F. Martinez, K. Okoshi, M.P. Okoshi, N-Acetylcysteine Influence on Oxidative Stress and Cardiac Remodeling in Rats During Transition from Compensated Left Ventricular Hypertrophy to Heart Failure, Cell Physiol Biochem 44(6) (2017) 2310-2321.

[110] T. Wilder, D.M. Ryba, D.F. Wieczorek, B.M. Wolska, R.J. Solaro, N-acetylcysteine reverses diastolic dysfunction and hypertrophy in familial hypertrophic cardiomyopathy, Am J Physiol Heart Circ Physiol 309(10) (2015) H1720-30.

[111] C. Rocca, T. Pasqua, L. Boukhzar, Y. Anouar, T. Angelone, Progress in the emerging role of selenoproteins in cardiovascular disease: focus on endoplasmic reticulum-resident selenoproteins, Cell Mol Life Sci 76(20) (2019) 3969-3985.

[112] A. Greasley, Y. Zhang, B. Wu, Y. Pei, N. Belzile, G. Yang, H2S Protects against Cardiac Cell Hypertrophy through Regulation of Selenoproteins, Oxid Med Cell Longev 2019 (2019) 6494306.

[113] P. Huang, Z. Shen, W. Yu, Y. Huang, C. Tang, J. Du, H. Jin, Hydrogen Sulfide Inhibits High-Salt DietInduced Myocardial Oxidative Stress and Myocardial Hypertrophy in Dahl Rats, Front Pharmacol 8 (2017) 128.

[114] J. Zhang, J. Yu, Y. Chen, L. Liu, M. Xu, L. Sun, H. Luo, Y. Wang, G. Meng, Exogenous Hydrogen Sulfide Supplement Attenuates Isoproterenol-Induced Myocardial Hypertrophy in a Sirtuin 3Dependent Manner, Oxid Med Cell Longev 2018 (2018) 9396089.

[115] G. Meng, J. Liu, S. Liu, Q. Song, L. Liu, L. Xie, Y. Han, Y. Ji, Hydrogen sulfide pretreatment improves mitochondrial function in myocardial hypertrophy via a SIRT3-dependent manner, Br J Pharmacol 175(8) (2018) 1126-1145. 
[116] Y. Bei, L.L. Pan, Q. Zhou, C. Zhao, Y. Xie, C. Wu, X. Meng, H. Gu, J. Xu, L. Zhou, J.P.G. Sluijter, S. Das, B. Agerberth, J. Sun, J. Xiao, Cathelicidin-related antimicrobial peptide protects against myocardial ischemia/reperfusion injury, BMC Med 17(1) (2019) 42.

[117] X. Wang, L. Chen, X. Zhao, L. Xiao, S. Yi, Y. Kong, Y. Jiang, J. Zhang, A cathelicidin-related antimicrobial peptide suppresses cardiac hypertrophy induced by pressure overload by regulating IGFR1/PI3K/AKT and TLR9/AMPKalpha, Cell Death Dis 11(2) (2020) 96.

[118] A.A. Aubdool, P. Thakore, F. Argunhan, S.J. Smillie, M. Schnelle, S. Srivastava, K.M. Alawi, E. Wilde, J. Mitchell, K. Farrell-Dillon, D.A. Richards, G. Maltese, R.C. Siow, M. Nandi, J.E. Clark, A.M. Shah, A. Sams, S.D. Brain, A Novel alpha-Calcitonin Gene-Related Peptide Analogue Protects Against EndOrgan Damage in Experimental Hypertension, Cardiac Hypertrophy, and Heart Failure, Circulation 136(4) (2017) 367-383.

[119] D.M. Ryba, C.M. Warren, C.N. Karam, R.T. Davis, 3rd, S.A.K. Chowdhury, M.G. Alvarez, M. McCann, C.W. Liew, D.F. Wieczorek, P. Varga, R.J. Solaro, B.M. Wolska, Sphingosine-1-Phosphate Receptor Modulator, FTY720, Improves Diastolic Dysfunction and Partially Reverses Atrial Remodeling in a Tm-E180G Mouse Model Linked to Hypertrophic Cardiomyopathy, Circ Heart Fail 12(11) (2019) e005835.

[120] S.S. Davies, L.S. May-Zhang, O. Boutaud, V. Amarnath, A. Kirabo, D.G. Harrison, Isolevuglandins as mediators of disease and the development of dicarbonyl scavengers as pharmaceutical interventions, Pharmacol Ther 205 (2020) 107418.

[121] L. Shang, X. Weng, D. Wang, W. Yue, R. Mernaugh, V. Amarnath, E.K. Weir, S.C. Dudley, Y. Xu, M. Hou, Y. Chen, Isolevuglandin scavenger attenuates pressure overload-induced cardiac oxidative stress, cardiac hypertrophy, heart failure and lung remodeling, Free Radic Biol Med 141 (2019) 291-298.

[122] G.K. Raut, S. Manchineela, M. Chakrabarti, C.K. Bhukya, R. Naini, A. Venkateshwari, V.D. Reddy, J.J. Mendonza, Y. Suresh, P. Nallari, M.P. Bhadra, Imine stilbene analog ameliorate isoproterenolinduced cardiac hypertrophy and hydrogen peroxide-induced apoptosis, Free Radic Biol Med 153 (2020) 80-88.

[123] T.M. Lee, H.J. Harn, T.W. Chiou, M.H. Chuang, C.H. Chen, C.H. Chuang, P.C. Lin, S.Z. Lin, Remote transplantation of human adipose-derived stem cells induces regression of cardiac hypertrophy by regulating the macrophage polarization in spontaneously hypertensive rats, Redox Biol 27 (2019) 101170.

[124] R. Amin, I. Muthuramu, J.P. Aboumsallem, M. Mishra, F. Jacobs, B. De Geest, Selective HDLRaising Human Apo A-I Gene Therapy Counteracts Cardiac Hypertrophy, Reduces Myocardial Fibrosis, and Improves Cardiac Function in Mice with Chronic Pressure Overload, Int J Mol Sci 18(9) (2017).

[125] Y. Zhang, J. Xu, Z. Long, C. Wang, L. Wang, P. Sun, P. Li, T. Wang, Hydrogen (H2) Inhibits Isoproterenol-Induced Cardiac Hypertrophy via Antioxidative Pathways, Front Pharmacol 7 (2016) 392.

[126] D.A. Guimaraes, M.A. Dos Passos, E. Rizzi, L.C. Pinheiro, J.H. Amaral, R.F. Gerlach, M.M. Castro, J.E. Tanus-Santos, Nitrite exerts antioxidant effects, inhibits the mTOR pathway and reverses hypertension-induced cardiac hypertrophy, Free Radic Biol Med 120 (2018) 25-32.

[127] Y. Zhang, W. Yan, X. Ji, H. Yue, G. Li, N. Sang, Maternal NO2 exposure induces cardiac hypertrophy in male offspring via ROS-HIF-1alpha transcriptional regulation and aberrant DNA methylation modification of Csx/Nkx2.5, Arch Toxicol 92(4) (2018) 1563-1579.

[128] S. Ebrahim, F.C. Taylor, P. Brindle, Statins for the primary prevention of cardiovascular disease, Bmj 348 (2014) g280.

[129] S. Kudo, K. Satoh, M. Nogi, K. Suzuki, S. Sunamura, J. Omura, N. Kikuchi, R. Kurosawa, T. Satoh, T. Minami, S. Ikeda, S. Miyata, H. Shimokawa, SmgGDS as a Crucial Mediator of the Inhibitory Effects of Statins on Cardiac Hypertrophy and Fibrosis: Novel Mechanism of the Pleiotropic Effects of Statins, Hypertension 67(5) (2016) 878-89.

[130] L. Xu, Y. Su, Y. Zhao, X. Sheng, R. Tong, X. Ying, L. Gao, Q. Ji, Y. Gao, Y. Yan, A. Yuan, F. Wu, F. Lan, J. $\mathrm{Pu}$, Melatonin differentially regulates pathological and physiological cardiac hypertrophy: Crucial role of circadian nuclear receptor RORalpha signaling, J Pineal Res 67(2) (2019) e12579. 
[131] A. Picca, G. Sirago, V. Pesce, A.M.S. Lezza, R. Calvani, M. Bossola, E.R. Villani, F. Landi, C. Leeuwenburgh, R. Bernabei, C.S. Carter, E. Marzetti, Administration of Enalapril Started Late in Life Attenuates Hypertrophy and Oxidative Stress Burden, Increases Mitochondrial Mass, and Modulates Mitochondrial Quality Control Signaling in the Rat Heart, Biomolecules 8(4) (2018).

[132] C. Zhang, F. Wang, Y. Zhang, Y. Kang, H. Wang, M. Si, L. Su, X. Xin, F. Xue, F. Hao, L. Yu, J. Xu, Y. Liu, M. Xue, Celecoxib prevents pressure overload-induced cardiac hypertrophy and dysfunction by inhibiting inflammation, apoptosis and oxidative stress, J Cell Mol Med 20(1) (2016) 116-27.

[133] A. Balkrishna, Y. Rustagi, K. Bhattacharya, A. Varshney, Application of Zebrafish Model in the Suppression of Drug-Induced Cardiac Hypertrophy by Traditional Indian Medicine Yogendra Ras, Biomolecules 10(4) (2020).

[134] P. Robless, D.P. Mikhailidis, G.P. Stansby, Cilostazol for peripheral arterial disease, Cochrane Database Syst Rev (1) (2008) Cd003748.

[135] S.Y. Wang, X. Ni, K.Q. Hu, F.L. Meng, M. Li, X.L. Ma, T.T. Meng, H.H. Wu, D. Ge, J. Zhao, Y. Li, G.H. $\mathrm{Su}$, Cilostazol alleviate nicotine induced cardiomyocytes hypertrophy through modulation of autophagy by CTSB/ROS/p38MAPK/JNK feedback loop, Int J Biol Sci 16(11) (2020) 2001-2013.

[136] X. Fang, M.J. Stroud, K. Ouyang, L. Fang, J. Zhang, N.D. Dalton, Y. Gu, T. Wu, K.L. Peterson, H.D. Huang, J. Chen, N. Wang, Adipocyte-specific loss of PPARgamma attenuates cardiac hypertrophy, JCI Insight 1(16) (2016) e89908.

[137] Y. Ma, W. Lv, Y. Gu, S. Yu, 1-Deoxynojirimycin in Mulberry (Morus indica L.) Leaves Ameliorates Stable Angina Pectoris in Patients With Coronary Heart Disease by Improving Antioxidant and Antiinflammatory Capacities, Front Pharmacol 10 (2019) 569.

[138] T. Jiang, X. Xing, L. Zhang, Z. Liu, J. Zhao, X. Liu, Chitosan Oligosaccharides Show Protective Effects in Coronary Heart Disease by Improving Antioxidant Capacity via the Increase in Intestinal Probiotics, Oxid Med Cell Longev 2019 (2019) 7658052.

[139] R.B. Singh, J. Fedacko, V. Mojto, D. Pella, Coenzyme Q10 Modulates Remodeling Possibly by Decreasing Angiotensin-Converting Enzyme in Patients with Acute Coronary Syndrome, Antioxidants (Basel) 7(8) (2018).

[140] Y. Li, H. Zhang, Soybean isoflavones ameliorate ischemic cardiomyopathy by activating Nrf2mediated antioxidant responses, Food Funct 8(8) (2017) 2935-2944.

[141] S.K. Maulik, V. Wilson, S. Seth, B. Bhargava, P. Dua, S. Ramakrishnan, C.K. Katiyar, Clinical efficacy of water extract of stem bark of Terminalia arjuna (Roxb. ex DC.) Wight \& Arn. in patients of chronic heart failure: a double-blind, randomized controlled trial, Phytomedicine 23(11) (2016) 1211-9.

[142] S.A. Mortensen, F. Rosenfeldt, A. Kumar, P. Dolliner, K.J. Filipiak, D. Pella, U. Alehagen, G. Steurer, G.P. Littarru, Q.S.S. Investigators, The effect of coenzyme Q10 on morbidity and mortality in chronic heart failure: results from Q-SYMBIO: a randomized double-blind trial, JACC Heart Fail 2(6) (2014) 641-9.

[143] J.E. Karlsson, W. El-Saadi, M. Ali, W. Puskar, P. Skogvard, J.E. Engvall, R.G. Andersson, E. Maret, P. Jynge, Mangafodipir as a cardioprotective adjunct to reperfusion therapy: a feasibility study in patients with ST-segment elevation myocardial infarction, Eur Heart J Cardiovasc Pharmacother 1(1) (2015) 39-45.

[144] S. Rautiainen, E.B. Levitan, N. Orsini, A. Akesson, R. Morgenstern, M.A. Mittleman, A. Wolk, Total antioxidant capacity from diet and risk of myocardial infarction: a prospective cohort of women, Am J Med 125(10) (2012) 974-80.

[145] M.L. McCullough, J.J. Peterson, R. Patel, P.F. Jacques, R. Shah, J.T. Dwyer, Flavonoid intake and cardiovascular disease mortality in a prospective cohort of US adults, Am J Clin Nutr 95(2) (2012) 45464.

[146] C.U. Chae, C.M. Albert, M.V. Moorthy, I.M. Lee, J.E. Buring, Vitamin E supplementation and the risk of heart failure in women, Circ Heart Fail 5(2) (2012) 176-82.

[147] D. Greig, H. Alcaino, P.F. Castro, L. Garcia, H.E. Verdejo, M. Navarro, R. Lopez, R. Mellado, F. Tapia, L.A. Gabrielli, C. Nogerol, M. Chiong, I. Godoy, S. Lavandero, Xanthine-oxidase inhibitors and 
statins in chronic heart failure: effects on vascular and functional parameters, J Heart Lung Transplant 30(4) (2011) 408-13. 


\begin{tabular}{|c|c|c|c|c|c|c|c|}
\hline Study type & Disease & $\begin{array}{l}\text { Study participants } \\
\text { (intervention group) }\end{array}$ & Intervention & Follow-up & Outcomes & Result & Reference \\
\hline $\begin{array}{l}\text { Randomised } \\
\text { clinical trial }\end{array}$ & $\mathrm{CHD}$ & $144(72)$ & $\begin{array}{l}\text { 1-Deoxynojirimycin } \\
\text { (Mulberry) }\end{array}$ & 4 weeks & $\begin{array}{l}\text { Increased LVEF and reduced } \\
\text { LVMI, aortic distensibility and } \\
\text { atherosclerosis index }(p< \\
0.05)\end{array}$ & Positive & [137] \\
\hline $\begin{array}{l}\text { Randomised } \\
\text { clinical trial }\end{array}$ & $\mathrm{CHD}$ & $110(56)$ & $\begin{array}{l}\text { Chitosan } \\
\text { Oligosaccharides }\end{array}$ & 6 months & $\begin{array}{l}\text { Improved Revised Cardiac } \\
\text { Risk Index scores, quality of } \\
\text { life scores and LVEF values }\end{array}$ & Positive & [138] \\
\hline $\begin{array}{l}\text { Randomised } \\
\text { clinical trial }\end{array}$ & AMI & $144(73)$ & Coenzyme Q10 & 24 weeks & $\begin{array}{l}\text { Attenuated LV remodelling: } \\
\text { decreased wall thickness at } \\
\text { and opposite the infarct site, } \\
\text { decreased LVM, reduced end } \\
\text { diastolic and systolic volumes }\end{array}$ & Positive & [139] \\
\hline $\begin{array}{l}\text { Randomised } \\
\text { clinical trial }\end{array}$ & $\mathrm{HCM}$ & $42(29)$ & N-Acetylcysteine & 12 months & $\begin{array}{l}\text { Small effect on indices of } \\
\text { cardiac hypertrophy: clinical } \\
\text { phenotype, } \\
\text { echocardiographic, and } \\
\text { cardiac magnetic resonance } \\
\text { imaging, function }\end{array}$ & Neutral & [108] \\
\hline $\begin{array}{l}\text { Randomised } \\
\text { clinical trial }\end{array}$ & IC & $200(100)$ & $\begin{array}{l}\text { Soybean } \\
\text { isoflavones }\end{array}$ & 24 weeks & $\begin{array}{l}\text { Associated with the reduction } \\
\text { of flow-mediated dilatation } \\
\text { impairment at } 24 \text { weeks (odds } \\
\text { ratio } 0.30,95 \% \mathrm{Cl} 0.14-0.85 \text {, } \\
\mathrm{P}=0.01 \text { ) }\end{array}$ & Positive & {$[140]$} \\
\hline $\begin{array}{l}\text { Randomised } \\
\text { clinical trial }\end{array}$ & $\mathrm{HF}$ & $100(50)$ & Arjuna extract & 12 weeks & $\begin{array}{l}\text { No change in LVEF }(24.3 \pm \\
7.1 \text { versus } 25.5 \pm 7.7 \% ; p= \\
0.4) \text { or secondary outcomes } \\
\text { (NYHA functional class level, } \\
\text { distance covered in } 6 \text { min } \\
\text { walk test, quality of life } \\
\text { scores) }\end{array}$ & Neutral & [141] \\
\hline
\end{tabular}




\begin{tabular}{|c|c|c|c|c|c|c|c|}
\hline $\begin{array}{l}\text { Randomised } \\
\text { clinical trial }\end{array}$ & $\mathrm{HF}$ & $420(202)$ & Coenzyme Q10 & 2 years & $\begin{array}{l}\text { The primary long-term } \\
\text { endpoint (MACE) was } \\
\text { reached by } 15 \% \text { of patients in } \\
\text { the intervention group (HR: } \\
0.50 ; 95 \% \mathrm{Cl}: 0.32 \text { to } 0.80 ; \\
p=0.003 \text { ). CoQ10 group also } \\
\text { had low cardiovascular } \\
\text { mortality and incidence of } \\
\text { hospital stays for HF }\end{array}$ & Positive & [142] \\
\hline $\begin{array}{l}\text { Randomised } \\
\text { clinical trial }\end{array}$ & STEMI & $20(10)$ & $\begin{array}{l}\text { Manganese } \\
\text { dipyridoxyl } \\
\text { diphosphate } \\
\text { (MnDPDP, } \\
\text { catalytic) }\end{array}$ & 48 hours & $\begin{array}{l}\text { Failed to decrease plasma } \\
\text { biomarker release, but } \\
\text { decreased the mean infarct } \\
\text { size and increased LVEF }\end{array}$ & Neutral & [143] \\
\hline $\begin{array}{l}\text { Prospective } \\
\text { cohort study }\end{array}$ & CVD & 32,561 (all women) & $\begin{array}{l}\text { Antioxidant- } \\
\text { containing foods }\end{array}$ & 10 years & $\begin{array}{l}\text { Myocardial infarction hazard } \\
\text { ratio for women comparing } \\
\text { the highest quintile of dietary } \\
\text { total antioxidant capacity to } \\
\text { the lowest was } 0.80 \text { ( } 95 \% \mathrm{Cl} \text {, } \\
0.67-0.97 ; p \text {-trend }=0.02) \text {, but } \\
\text { with no significant inverse } \\
\text { association }\end{array}$ & Neutral & [144] \\
\hline $\begin{array}{l}\text { Prospective } \\
\text { cohort study }\end{array}$ & CVD & $\begin{array}{l}98,469(60,289 \\
\text { women) }\end{array}$ & Flavonoid & 7 years & $\begin{array}{l}\text { Men and women with total } \\
\text { flavonoid intakes in the top } \\
\text { quintile had a lower risk of } \\
\text { fatal CVD (RR: } 0.82 ; 95 \% \mathrm{Cl} \text { : } \\
0.73,0.92 ; \mathrm{p} \text {-trend }=0.01 \text { ). } \\
\text { Five flavonoid classes } \\
\text { (anthocyanidins, flavan-3-ols, } \\
\text { flavones, flavonols, } \\
\text { proanthocyanidins) were } \\
\text { individually associated with } \\
\text { lower risk of fatal CVD }\end{array}$ & Positive & [145] \\
\hline $\begin{array}{l}\text { Randomised } \\
\text { clinical trial }\end{array}$ & $\mathrm{HF}$ & $\begin{array}{l}39,815(19,913, \text { all } \\
\text { women) }\end{array}$ & Vitamin E & 10 years & $\begin{array}{l}\text { No significant effect on HF } \\
\text { risk (HR } 0.93 ; 95 \% \mathrm{Cl}, 0.71-\end{array}$ & Neutral & [146] \\
\hline
\end{tabular}




\begin{tabular}{|c|c|c|c|c|c|c|c|}
\hline & & & & & $\begin{array}{l}1.21 ; p=0.59) \text {. Vitamin } \mathrm{E} \text { was } \\
\text { inversely related to } \\
\text { development of } \mathrm{HFpEF}(\mathrm{HR} \\
0.59 ;(95 \% \mathrm{Cl}, 0.38-0.92 ; \\
\mathrm{p}=0.02), \text { but there was no } \\
\text { statistically significant effect } \\
\text { on the risk of developing } \\
\text { systolic HF }(\mathrm{HR} 1.26 ; 95 \% \mathrm{Cl} \text {, } \\
0.84-1.89 ; \mathrm{p}=0.26)\end{array}$ & & \\
\hline $\begin{array}{l}\text { Randomised } \\
\text { clinical trial }\end{array}$ & $\mathrm{HF}$ & 74 (38) & Allopurinol & 4 weeks & $\begin{array}{l}\text { No additional beneficial } \\
\text { effects of alopurinol were } \\
\text { found after completion with } \\
\text { the short-term atorvastatin } \\
\text { treatment in HF patients }\end{array}$ & Neutral & [147] \\
\hline
\end{tabular}


Figure 1: Schematic indicating oxidative stress pathways regulated by NOX isoforms with potential points of intervention (Red boxes- detrimental cascades; green boxes- protective cascades)

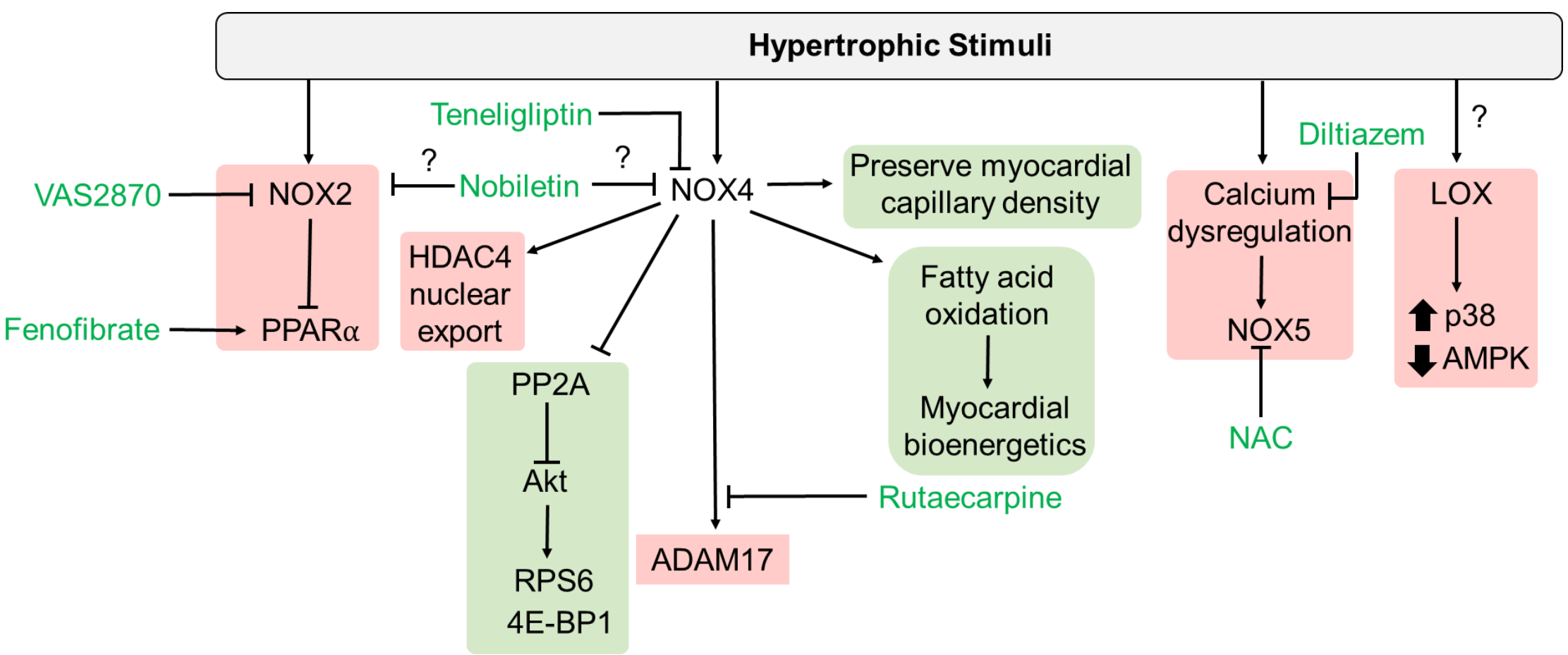


Figure 2: Schematic indicating oxidative stress pathways in response to metabolic disturbances with potential points of intervention (Red boxesdetrimental cascades; green boxes- protective cascades; ?- further validation required)

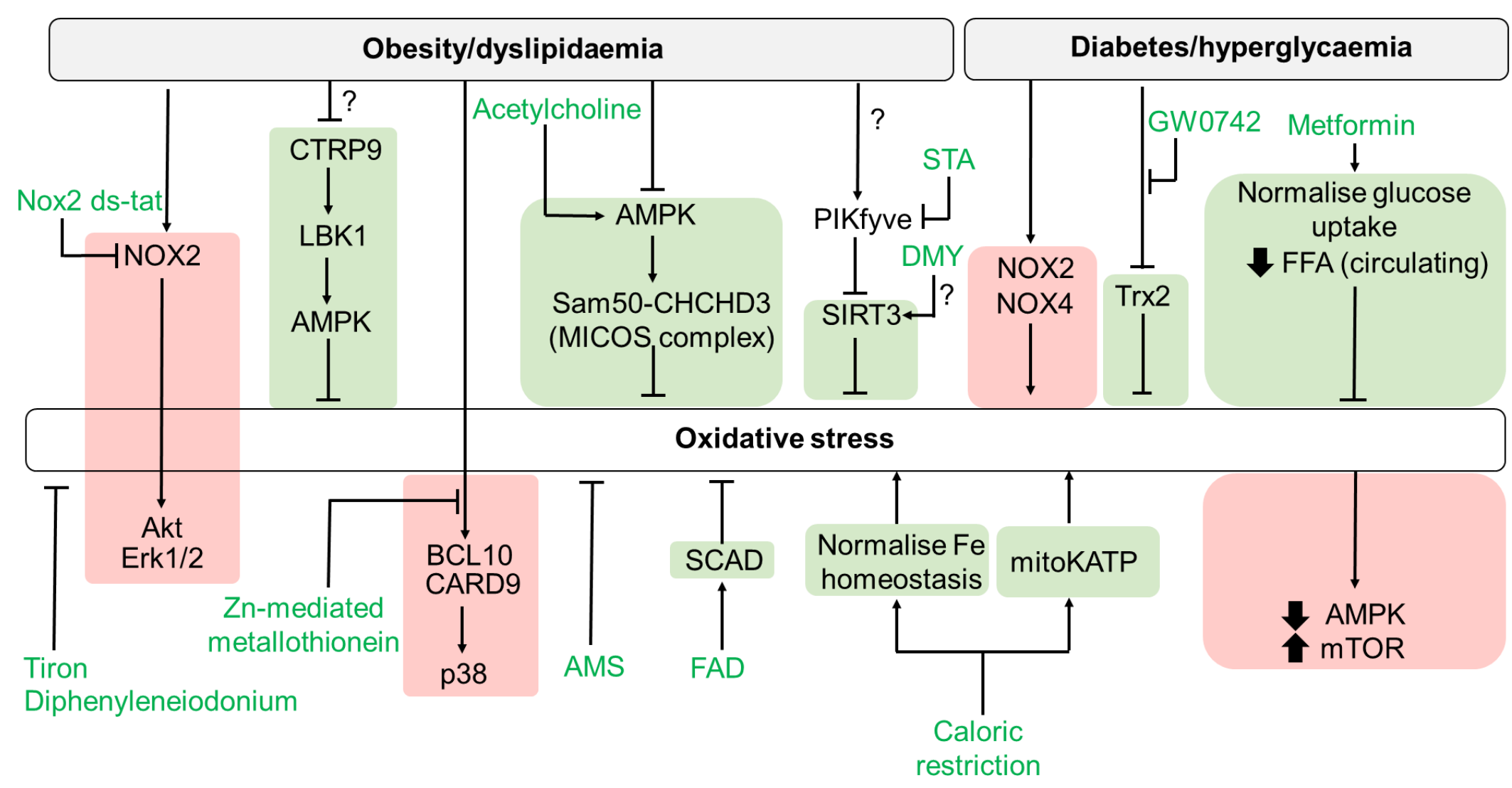


Figure 3: Schematic indicating oxidative stress pathways in response to mitochondrial dysfunction with potential points of intervention (Red boxes- detrimental cascades; green boxes- protective cascades; ?- further validation required).

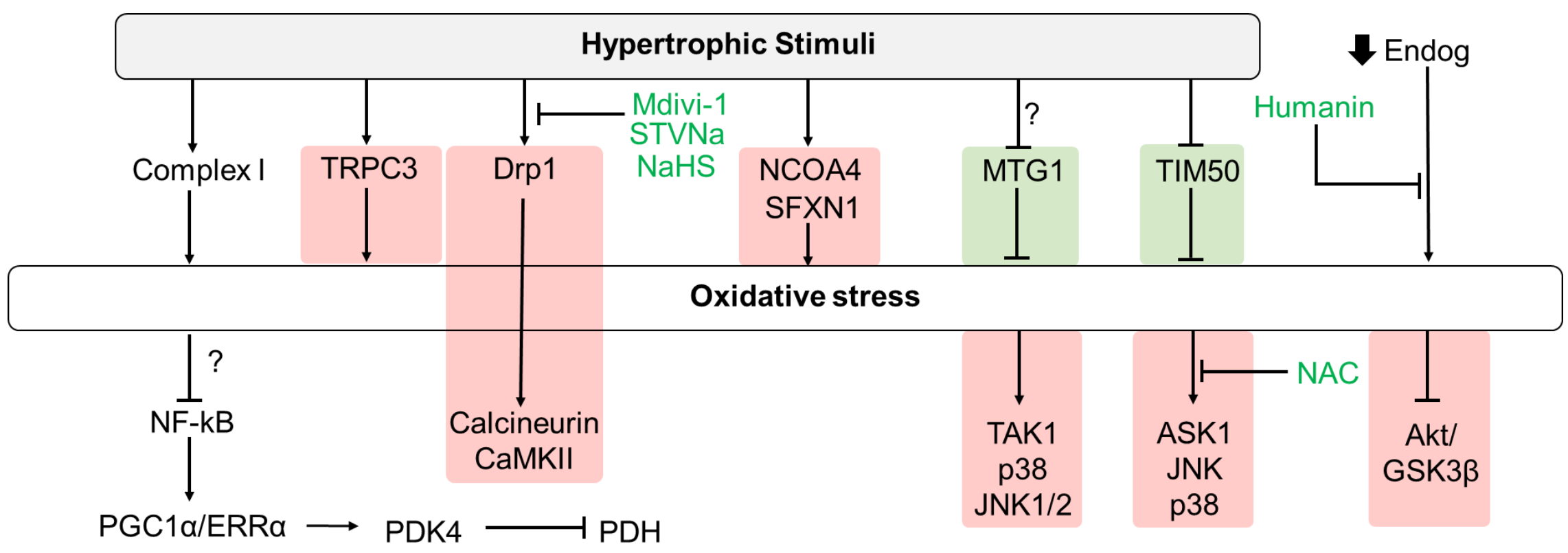


Figure 4: Schematic indicating oxidative stress pathways in response to inflammation with potential points of intervention (Red boxes- detrimental cascades; green boxes- protective cascades; ?- further validation required).

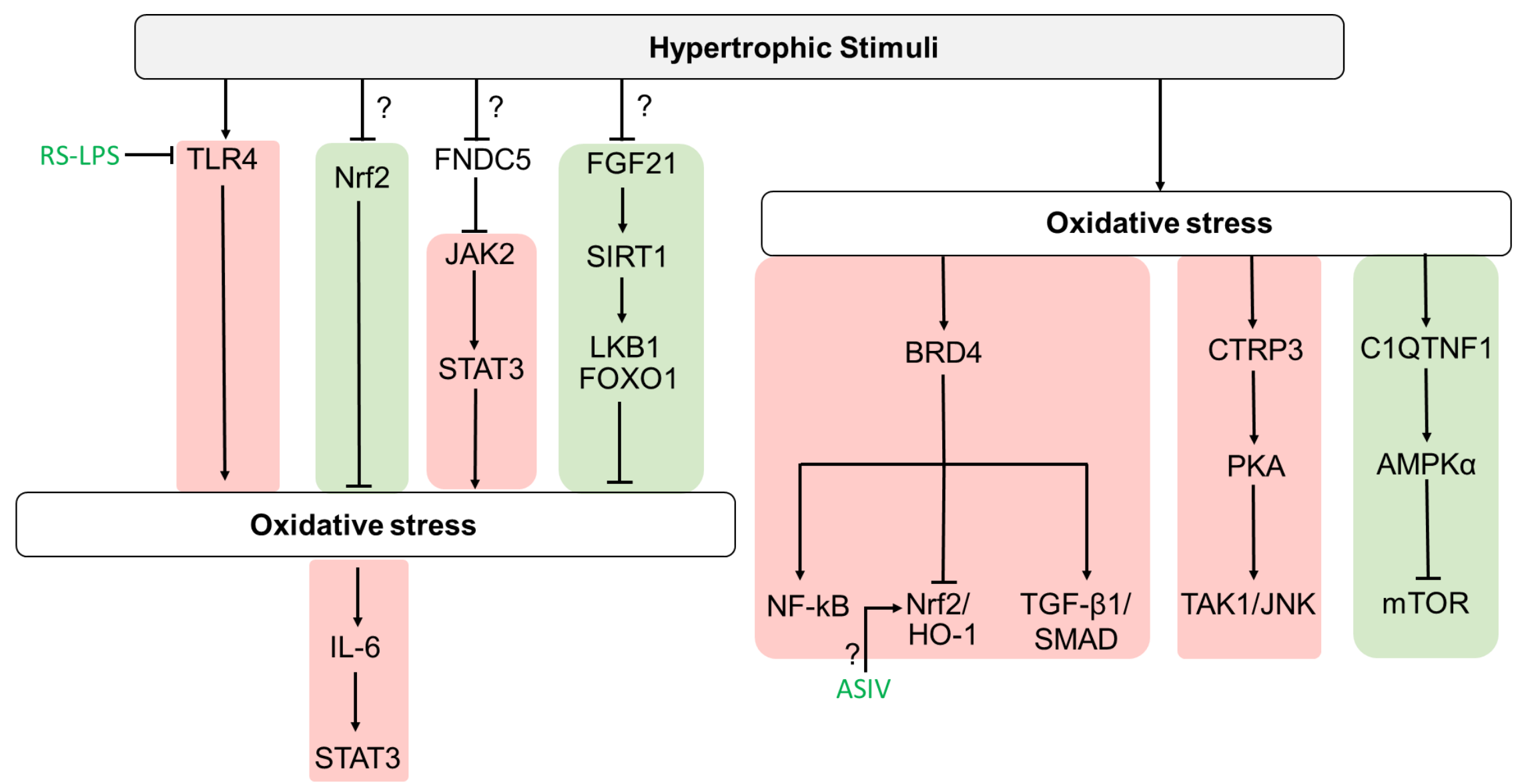


Figure 5: Schematic indicating oxidative stress pathways in response to dysregulated cellular processes (impaired/excessive autophagy, dysregulated protein quality control and miRNAs) with potential points of intervention (Red boxes- detrimental cascades; green boxes- protective cascades; ?- further validation required).

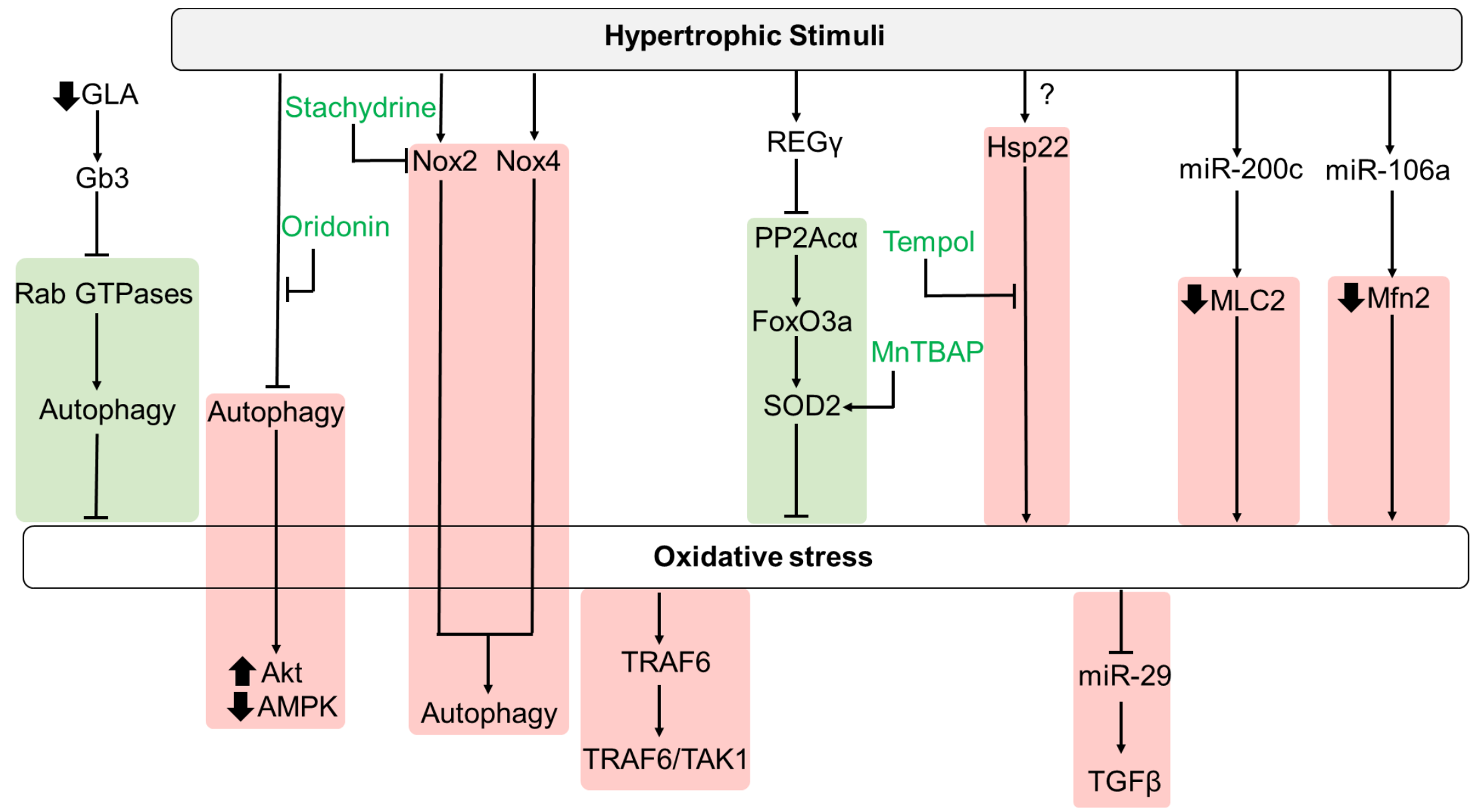


Figure 6: Schematic indicating oxidative stress pathways in response to impaired ion channel activity with potential points of intervention (Red boxes- detrimental cascades; green boxes- protective cascades; ?- further validation required).

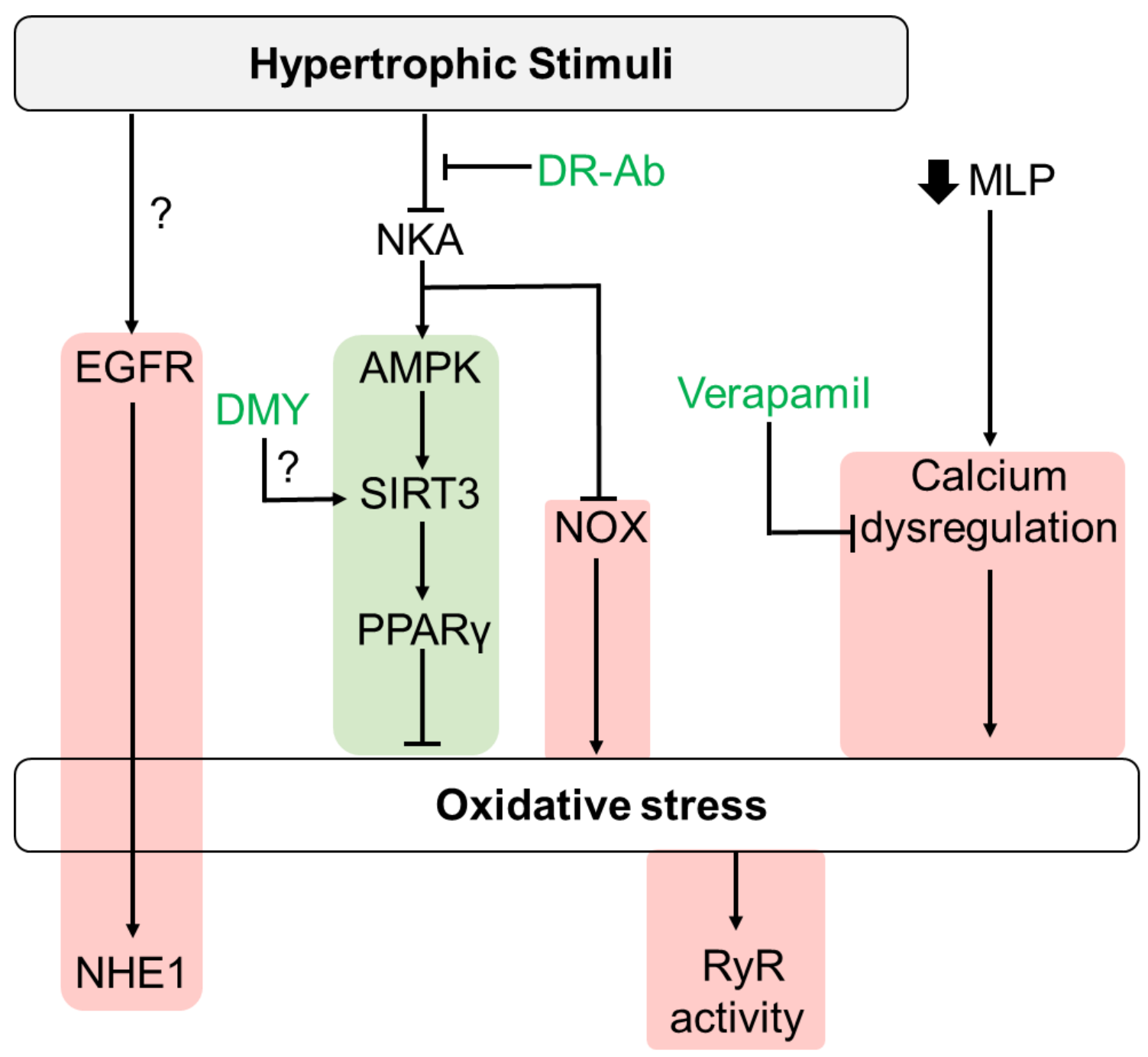

